\title{
Per l'edizione critica della rime di Baldassarre Castiglione e Cesare Gonzaga. Censimento e questioni attributive*
}

\section{Matteo Fadini}

\begin{abstract}
Riassunto: Larticolo offre i risultati del primo censimento dei testimoni delle rime di B. Castiglione e C. Gonzaga. Alla luce della tradizione vengono affrontate le questioni attributive riguardanti i testi poetici: prima del presente intervento le rime ritenute castiglionesche erano $42^{1}$ - tra le quali Euro gentil che in tempi recenti è stato definitivamente attribuito a Ercole Strozzi — mentre i testi gonzaghiani editi si limitavano a una canzone, un capitolo ternario e un sonetto. I risultati del censimento e l'analisi della tradizione suggeriscono di togliere a Castiglione 8 testi e assegnarli ad altri autori, rispettivamente Luigi Cassola (5), Giacomo Bonfadio (2) e Girolamo Volpe (1); per altri 3 testi si avanza l'ipotesi che siano attribuibili a Gonzaga e non a Castiglione. Di conseguenza, il corpus gonzaghiano conta 10 componimenti, di cui 1 già prima stampato tra le rime dubbie di Bembo. Infine si fornisce l'edizione critica di 2 componimenti: Superbi colli, e voi sacre ruine e Io dico: Amor, chi dà tal vena.
\end{abstract}

\section{Censimento delle rime di Castiglione e Gonzaga}

I testimoni manoscritti sono individuati con sigle alfabetiche riferite ai luoghi ed eventualmente agli enti di conservazione (ulteriori distinzioni sono affidate a esponenti numerici). ${ }^{2}$

\footnotetext{
* Il saggio è frutto della rielaborazione di una parte della tesi di laurea specialistica dal titolo Per l'edizione critica delle rime di Baldassarre Castiglione e Cesare Gonzaga (relatore Andrea Comboni, correlatore Nello Bertoletti), discussa nel luglio del 2010 presso la Facoltà di Lettere e Filosofia di Trento.

${ }^{1}$ Dal computo sono escluse l'egloga Tirsi scritta a quattro mani con il cugino e la ballata Queste lagrime mie contenuta nella stessa egloga.

${ }^{2}$ Di fatto il criterio adottato è molto simile a quello utilizzato da Donnini per la sua edizione delle rime di Bembo.
} 
Per quanto riguarda i testimoni a stampa, quelli contenenti esclusivamente o prevalentemente componimenti castiglioneschi sono stati indicati con la prima parola del titolo in corsivo, gli altri con il solo anno di pubblicazione in grassetto. Si è deciso di non tenere conto delle stampe musicali poiché esse risultano poco utili ai fini ecdotici e ancor meno in relazione alla paternità dei testi poetici. ${ }^{3}$

Per ragioni di spazio non si forniranno le descrizioni dei testimoni né la bibliografia relativa.

\subsection{Elenco dei manoscritti}

Ba Bassano del Grappa, Biblioteca Comunale, 29.B.8.

BI Bologna, Archivio Cavazza-Isolani, F. 69/166 (ora in CN 95).

BA $A_{1}$ Bologna, Biblioteca Comunale dell'Archiginnasio, A. 2044-2045.

BA 2 Bologna, Biblioteca Comunale dell'Archiginnasio, A. 2429.

BA $A_{3}$ Bologna, Biblioteca Comunale dell'Archiginnasio, A. 2646 - fasc.II.

BU $_{1}$ Bologna, Biblioteca Universitaria, 1250.

BU $_{2}$ Bologna, Biblioteca Universitaria, 2620.

BU $_{3}$ Bologna, Biblioteca Universitaria, 3797.

$\mathbf{B U}_{4}$ Bologna, Biblioteca Universitaria, 4052 - fasc. 14.

Fe Ferrara, Biblioteca Comunale Ariostea, II 449.

FL Firenze, Biblioteca Medicea Laurenziana, Plut. XLI.25.

$\mathbf{F M}_{1}$ Firenze, Biblioteca Moreniana, 256.

$\mathbf{F M}_{2}$ Firenze, Biblioteca Moreniana, 258.

FN $_{1}$ Firenze, Biblioteca Nazionale Centrale, Magl. VII 719.

$\mathbf{F N}_{2}$ Firenze, Biblioteca Nazionale Centrale, Magl. VII 720.

$\mathbf{F N}_{3}$ Firenze, Biblioteca Nazionale Centrale, Magl. VII 727.

$\mathbf{F N}_{4}$ Firenze, Biblioteca Nazionale Centrale, Magl. VII 1041.

FN $_{5}$ Firenze, Biblioteca Nazionale Centrale, Magl. VII 1192.

$\mathbf{F N}_{6}$ Firenze, Biblioteca Nazionale Centrale, Magl. VII 1403.

$\mathbf{F N}_{7}$ Firenze, Biblioteca Nazionale Centrale, II.I.60 (già Magl. VII 724).

$\mathbf{F N}_{8}$ Firenze, Biblioteca Nazionale Centrale, Palat. 221.

FN, Firenze, Biblioteca Nazionale Centrale, Palat. 288.

FR Firenze, Biblioteca Riccardiana, 2803.

Fo Foligno, Biblioteca L. Jacobilli del Seminario vescovile, B V 8.

Lu Lucca, Biblioteca Statale, 1328 (=L55).

\footnotetext{
${ }^{3}$ Segnalo che almeno 16 stampe musicali del XVI secolo contengono testi castiglioneschi.
} 
Mi 1 Milano, Biblioteca Ambrosiana, H 53 inf.

Mi 2 Milano, Biblioteca Ambrosiana, R 105 sup.

Mo Modena, Biblioteca Estense Universitaria, a .T.9.19 (Ital. 838).

Na Napoli, Biblioteca Statale del Monumento Nazionale dei Gerolamini, M. XXVIII 1-8.

$\mathbf{P d}_{1}$ Padova, Biblioteca del Seminario Vescovile, 91.

$\mathbf{P d}_{2}$ Padova, Biblioteca del Seminario Vescovile, 163.

Pr Parma, Biblioteca Palatina, Palatino 557.

Pv Pavia, Biblioteca Universitaria, Aldini 156.

Pc Piacenza, Biblioteca Comunale Passerini-Landi, ms. Pallastrelli 230.

RC Roma, Biblioteca Casanatense, 897.

RN Biblioteca Nazionale Centrale, Fondo Gesuitico 291.

Gc Santa Monica, Getty Center for History of Arts and the Humanities, 850626.

$\mathbf{S i}_{1}$ Siena, Biblioteca Comunale degli Intronati, H X 38.

$\mathbf{S i}_{2}$ Siena, Biblioteca Comunale degli Intronati, I VI 41.

Td Todi, Biblioteca Comunale Lorenzo Leoni, 238.

UB Udine, Biblioteca Arcivescovile e Bartoliniana, bart. 45.

UC Udine, Biblioteca Comunale Vincenzo Joppi, 286.

$\mathbf{V}_{2}$ Città del Vaticano, Biblioteca Apostolica Vaticana, Vat. Lat. 3351.

$\mathbf{V}_{3}$ Città del Vaticano, Biblioteca Apostolica Vaticana, Vat. Lat. 5170.

$\mathbf{V}_{4}$ Città del Vaticano, Biblioteca Apostolica Vaticana, Vat. Lat. 5182.

$\mathbf{V}_{5}$ Città del Vaticano, Biblioteca Apostolica Vaticana, Vat. Lat. 5187.

$\mathbf{V}_{6}$ Città del Vaticano, Biblioteca Apostolica Vaticana, Vat. Lat. 5225 (voll. I e II).

$\mathbf{V}_{7}$ Città del Vaticano, Biblioteca Apostolica Vaticana, Vat. Lat. 5226 (vol. I).

$\mathbf{V}_{9}$ Città del Vaticano, Biblioteca Apostolica Vaticana, Vat. Lat. 8203.

$\mathbf{V}_{10}$ Città del Vaticano, Biblioteca Apostolica Vaticana, Vat. Lat. 8856,

$\mathbf{V}_{12}$ Città del Vaticano, Biblioteca Apostolica Vaticana, Vat. Lat. 9948.

$\mathbf{V}_{13}$ Città del Vaticano, Biblioteca Apostolica Vaticana, Vat. Lat. 10286.

$\mathbf{V}_{14}$ Città del Vaticano, Biblioteca Apostolica Vaticana, Vat. Lat. 13704.

$\mathbf{V}_{15}$ Città del Vaticano, Biblioteca Apostolica Vaticana, Regin. Lat. 1591.

$\mathbf{V}_{16}$ Città del Vaticano, Biblioteca Apostolica Vaticana, Urb. Lat. 490.

$\mathbf{V}_{17}$ Città del Vaticano, Biblioteca Apostolica Vaticana, Barb. Lat. 3945.

$\mathbf{V}_{18}$ Città del Vaticano, Biblioteca Apostolica Vaticana, Rossiano 1003.

$\mathbf{V e}_{1}$ Venezia, Biblioteca Nazionale Marciana, Ita IV, 1795 (= 10653).

$\mathbf{V e}_{2}$ Venezia, Biblioteca Nazionale Marciana, Ita IX, 154 (= 6752).

$\mathbf{V e}_{3}$ Venezia, Biblioteca Nazionale Marciana, Ita. IX, 203 (= 6757) 
$\mathbf{V e}_{4}$ Venezia, Biblioteca Nazionale Marciana, Ita. XI, 66 (=6730).

Wi Wien, Österreichische Nationalbibliotek, Vind. Pal. 9952.

Wr Wrocłav, Biblioteka Uniwersytecka, Milich. Coll. IV 18 (Akz. 1951, 289 [18, 9350]).

\subsubsection{Manoscritti di rime erratamente attribuite a Castiglione}

BC $_{1}$ Bologna, Biblioteca di casa Carducci, 85.

$\mathbf{B C}_{2}$ Bologna, Biblioteca di casa Carducci, 90.

$\mathbf{V}_{8}$ Città del Vaticano, Biblioteca Apostolica Vaticana, Vat. Lat. 7547.

$\mathbf{V}_{1}$ Città del Vaticano, Biblioteca Apostolica Vaticana, Vat. Lat. 8860.

\subsubsection{Manoscritti non reperibili o non contenenti rime di Castiglione}

Kristeller segnala il manoscritto 68 presso i marchesi Capilupi a Suzzara descrivendolo così:

misc. XVI. Carmina by Franc. Conternius, Lazarus Bonamicus [...] Balthasar Castilionus, Alfonsus Capilupus, Jo. Bapt. Morellus. Also some Rime. ${ }^{4}$

Purtroppo non è stato possibile visionare questo manoscritto che risulta al momento irrintracciabile.

Sempre Kristeller segnala che il ms. Y 33 sup. della Biblioteca Ambrosiana di Milano conterrebbe rime di Castiglione, ${ }^{5}$ traendo l'informazione, errata, dall'inventario Ceruti. Il manoscritto in questione è un miscellaneo di rime, molte delle quali sono testi di corrispondenza. La maggior parte di questi testi è di Baldassarre Castoldi: molto probabilmente la rubrica a c. 29r "Baldassare Cast." (con 'i' soprascritta) deve aver tratto in inganno il bibliotecario dell'Ambrosiana e, con lui, anche Kristeller.

\footnotetext{
${ }^{4}$ Paul Oskar Kristeller (a cura di), Iter Italicum, 6 voll., London-Leiden, The Warburg Institute - E. J. Brill, 1967-1992, vol. II, p. 174.

${ }^{5}$ Ivi, vol. I, p. 316
} 
Infine il Carboni propone, anche se con riserve, ${ }^{6}$ di attribuire il sonetto Ligiadra donna del mio cor regina al Castiglione; questo testo si trova a c. $35 \mathrm{v} \mathrm{di}$ $\mathbf{V}_{1}{ }^{7}$ con l'attribuzione Baldassarre Estense e sembra non avere altre attestazioni. Il manoscritto in questione è un miscellaneo di $91 \mathrm{cc}$. contenente sia testi in latino di autori classici e umanistici sia liriche in volgare, la cui datazione al XV secolo non è mai stata messa in dubbio. Ragioni cronologiche spingono a non ritenere probabile la presenza di un testo castiglionesco in questo manoscritto, tanto più che un Baldassarre Estense è esistito: si tratta di un pittore, figlio naturale di Niccolò III d'Este, nato a Reggio Emilia attorno al $1441 .{ }^{8}$ Baldassarre Estense non risulta autore di testi letterari, ma non sarebbe il primo caso di pittore-poeta; in ogni caso, al testo in questione non pare di dover accostare il nome di Castiglione, pur rimanendo aperta la questione sulla paternità.

\subsection{Elenco delle stampe}

\subsubsection{Collettori}

Stanze $=$ Stanze pastorali, del conte Baldesar Castiglione, et del signor Cesare Gonzaga, con le rime di Anton Giacomo Corso, Venezia, eredi Aldo Manuzio, 1553.

Elogi $=$ Elogi historici di alcuni personaggi della famiglia Castigliona; gia raccolti da Antonio Beffa Negrini; et hora dati in luce da Francesco Osanna. Con sette tauole, contenenti vna quasi idea di tutta l'opera, Mantava, Francesco Osanna, 1606.

Opere $=$ Opere volgari, e latine del conte Baldessar Castiglione. Novellamente raccolte, ordinate, ricorrette, ed illustrate, come nella seguente lettera può vedersi, da Gio. Antonio, e Gaetano Volpi, Padova, Giuseppe Comino, 1733.

Poesie $=$ Poesie volgari, e latine del conte Baldessar Castiglione corrette, illustrate, ed accresciute di varie cose inedite aggiuntevi alcune rime e lettere di Cesare Gonzaga suo cugino, Roma, Niccolò e Marco Pagliarini, 1760.

Lettere $=$ Lettere del conte Baldessar Castiglione ora per la prima volta date in luce e con annotazioni storiche illustrate dall'abate Pierantonio Serassi. Volume primo [-secondo], Padova, Giuseppe Comino, 1769-1771.

\footnotetext{
${ }^{6} \mathrm{Nel}$ volume di indici dell'incipitario di Carboni s.v. Castiglione Baldassarre si trova il riferimento a questo ms., ma l'indicazione è in carattere corsivo, che segnala una variante particolare del nome o una attribuzione non certa.

${ }^{7}$ Vat. Lat. 2932.

${ }^{8}$ Edoardo Arlsan, "Baldassarre Estense", Dizionario Biografico degli Italiani, v (1963).
} 
Canzonieri = Ariosto. Castiglione. Fracastoro. Sanazzaro. Casa. Canzonieri del secolo XVI, Venezia, Antonio Zatta e figli, 1787.

\subsubsection{Stampe miscellanee}

1514 = Cancionero general de muchos y diversos autores, Valencia, Jorge Costilla, $1514 .^{9}$

1545 = Rime diuerse di molti eccellentiss. auttori nuouamente raccolte. Libro primo, Venezia, Gabriel Giolito, $1545 .^{10}$

1547 = Rime di diuersi nobili huomini et eccellenti poeti nella lingua thoscana. Libro secondo Venezia, Gabriel Giolito, $1547 .{ }^{11}$

$\mathbf{1 5 5 0}$ = Libro terzo de le rime di diuersi nobilissimi et eccellentissimi autori nuouamente raccolte, Venzia, al segno del Pozzo, 1550.

6-1553 = Il sesto libro delle rime di diuersi eccellenti autori, nuouamente raccolte, et mandate in luce. Con un discorso di Girolamo Ruscelli, Venezia, al segno del Pozzo, 1553.

1553 = Rime di diuersi eccellenti autori raccolte dai libri da noi altre volte impressi tra le quali se ne leggono molte non più vedute, Venezia, Gabriel Giolito e fratelli, $1553 .^{12}$

1560 = Rime di diuersi autori eccellentiss. Libro nono, Cremona, Vincenzo Conti, 1560.

\footnotetext{
9 Secondo edizione dell'opera di Hernando del Castillo; la princeps uscì nel 1511, sempre a Valencia, per i tipi di Christoph Kaufmann e non contiene il testo di Gonzaga presente a partire dalla ristampa del 1514 .

${ }^{10}$ Questo volume è stato ristampato due volte: Rime diuerse di molti eccellentiss. auttori nuouamente raccolte. Libro primo con nuoua additione ristampato, Venezia, Gabriel Giolito, 1546 e Rime diuerse di molti eccellentiss. auttori nuouamente raccolte. Libro primo con nuoua additione ristampato, Venezia, Gabriel Giolito, 1549.

${ }^{11}$ La ristampa dell'anno successivo ha un titolo leggermente differente: Delle rime di diuersi nobili huomini et eccellenti poeti nella lingua thoscana. Nuouamente ristampate. Libro secondo, Venezia, Gabriel Giolito, 1548.

${ }^{12}$ Ristampato nel 1556 col titolo: Rime di diuersi, et eccellenti autori, raccolte dai libri da noi altre volte impressi, tra le quali, se ne leggono molte non più vedute. Di nuouo ricorrette e ristampate, Venezia, Gabriel Giolito e fratelli, 1556.
} 
1563 = Il primo [-secondo] volume delle rime scelte da diuersi autori, di nuouo corrette, et ristampate, Venezia, Gabriel Giolito, $1563 .^{13}$

1565 = De le rime di diuersi nobili poeti toscani, raccolte da $m$. Dionigi Atanagi, libro secondo. Con vna tauola del medesimo, ne la quale, oltre molte altre cose degne di notitia, taluolta si dichiarano alcune cose pertinenti a la lingua toscana, et al'arte del poetare, Venetia, Lodovico Auanzo, 1565.

1573 = Scelta nuoua di rime, de' piu illustri, et eccellenti poeti dell'eta nostra, del s. Girolamo Ruscelli, Venezia, Giacomo Simbeni, 1573.

1739 = Scelta di sonetti e canzoni de' piu eccellenti rimatori d'ogni secolo [...] Parte prima. Volume primo, Venezia, Lorenzo Baseggio, 1739.

1846-7 = Poesie italiane inedite di dugento autori dall'origine della lingua infino al secolo decimosettimo raccolte e illustrate da Francesco Trucchi, Prato, Ranieri Guasti, 4 vol., 1846-1847, vol. III.

\subsubsection{Miscellanee di rime erratamente attribuite a Castiglione}

1551 = Libro quarto delle rime di diuersi eccellentiss. autori nella lingua volgare. Nouamente raccolte, Bologna, Anselmo Giaccarello, 1551.

1558 = I fiori delle rime de' poeti illustri, nuouamente raccolti et ordinati da Girolamo Ruscelli. Con alcune annotationi del medesimo, sopra i luoghi, che le ricercano per l'intendimento delle sentenze, o per le regole et precetti della lingua, et dell'ornamento, Venetia, Giovanni Battista e Melchiorre Sessa, 1558. ${ }^{14}$

1713 = Rime scelte de' poeti ferraresi antichi, e moderni. Aggiuntevi nel fine alcune brevi notizie istoriche intorno ad essi, Ferrara, eredi Bernardino Pomatelli, 1713.

\section{Risultati del censimento e storia della tradizione}

\subsection{Tradizione a stampa}

Le rime di Castiglione e Gonzaga non furono mai stampate viventi gli autori, né sono state mai raccolte organicamente in seguito. In tutto il complesso della

\footnotetext{
${ }^{13}$ Antologia di ampio successo, ristampata sei volte nel corso del secolo (1564, 1565, 1586, $1587,1588,1590)$ con minime modifiche nel titolo.

${ }^{14}$ Volume ripubblicato presso il medesimo stampatore nel 1569, 1579 e 1586.
} 
tradizione a stampa possiamo individuare tre insiemi di testimoni che verranno trattati separatamente in ragione della loro intrinseca differenza: ${ }^{15}$

1. le antologie;

2. le opere che organizzano la produzione poetica;

3. le pubblicazioni otto-novecentesche di inediti.

\subsubsection{Le antologie}

A partire dalle Rime diuerse di molti [...] Libro primo (= 1545) fino a Scelta di sonetti e canzoni (= 1739), si può dire che la maggior parte delle antologie di rime presenta almeno un testo di Castiglione o di Gonzaga. In totale sono $10 \mathrm{i}$ testi che si possono leggere in queste raccolte; li elenco ricordando che i primi otto sono di Baldassarre, gli ultimi due del cugino:

1. Queste lagrime mie, questi sospiri, ballata

2. Cantai mentre nel cor lieto fioria, sonetto

3. Euro gentil, che gli aurei crespi nodi, sonetto

4. Superbi colli, e voi sacre ruine, sonetto

5. Manca il fior giovenil de' miei prim'anni, canzone

6. L'alta catena, Amor, la fiamma ardente, sonetto

7. Quando il tempo, che 'l ciel con gli anni gira, sonetto

8. Ecco la bella fronte e il dolce nodo, sonetto

9. D'uno in altro desir quest'alma stanca, canzone

10. Amar stimol d'amor che hai l'alma acesa, capitolo ternario

I primi tre testi apparvero nel primo libro delle Rime diverse $(=\mathbf{1 5 4 5})$ e si trovano nello stesso ordine anche nelle due ristampe di questo libro uscite nel 1546 e nel $1549 .{ }^{16}$ Occorre ricordare che il primo componimento di questo gruppo, pur avendo una tradizione autonoma anche manoscritta, è in realtà una ballata recitata da Jole dopo la XXIX ottava del Tirsi: ragion per cui la si trova anche nelle successive stampe che tramandano l'intera egloga. Il terzo testo (Euro gentil)

\footnotetext{
${ }^{15}$ Come indicato supra, non si è tenuto conto delle stampe musicali.

${ }^{16}$ Non si considera la stampa Frottole libro undecimo, Fossombrone, Ottaviano Petrucci, 1514 dove si può leggere la ballata alle cc. $63 \mathrm{v}-64 \mathrm{r}$, musicata da Tromboncino, poiché si tratta, appunto, di una stampa musicale.
} 
è presente anche nel Libro quarto delle rime di diuersi $(=1551)$ con una variante importante nell'incipit (Lascivo euro) e con l'attribuzione a Ercole Strozzi. ${ }^{17}$

La princeps di Superbi colli è nel libro secondo delle Rime diverse (= 1547) e l'altra attestazione nelle antologie si trova nella ristampa del medesimo dell'anno successivo: in entrambi i testimoni la poesia è adespota. ${ }^{18}$

La canzone Manca il fior giovenil è il testo che ha avuto maggiore fortuna: la troviamo nel Libro terzo delle rime di diuersi $(=\mathbf{1 5 5 0})$, ne $\mathrm{Il}$ sesto libro delle rime di diuersi (= 6-1553) e in due «antologie di antologie» (1553 e 1563).

Il sesto libro (= 6-1553), appena citato, riporta anche il sonetto L'alta catena, Amor, mentre il nono libro $(=\mathbf{1 5 6 0})$ offre anch'esso una coppia di testi: Quando il tempo, che 'l ciel ed Ecco la bella fronte; l'ultima antonoligia cinqucentesca (1573) tramanda gli stessi due testi di 6-1553.

Nella più importante antologia del ' $700^{19}($ 1739) è presente un solo sonetto di Castiglione: Cantai mentre nel cor lieto fioria.

La tradizione delle rime di Gonzaga, d'altra parte, è molto più esigua. L'antologia De le rime di diversi nobili poeti toscani $(=1565)$ è la prima stampa ad attribuire esplicitamente una poesia a Cesare Gonzaga, la canzone D'uno in altro desir quest'alma stanca. Non è questa però la sola stampa antica utile per la ricostruzione critica dei testi gonzaghiani. A partire dalla seconda edizione del Cancionero general $(=\mathbf{1 5 1 4}),{ }^{20}$ monumentale opera di Hernando del Castillo che sistematizzò la produzione poetica spagnola del XVI sec., si legge una piccola sezione di poesie italiane; si tratta di cinque capitoli ternari:

1. Dolce mal, dolce guerra e dolce inganno;

2. Dolce et amar destin che me sospince [sic];

3. Amar stimol d'amor che hai l'alma acesa;

\footnotetext{
${ }^{17}$ Per l'analisi dei problemi attributivi di questo testo si veda la sezione 3.1.

${ }^{18}$ La discussione della paternità di questo testo è svolta infra, 3.2.

${ }^{19}$ Si tratta di una delle cinque antologie usufruite da Quondam per il suo studio sulla ricezione del petrarchismo: cfr. Amedeo Quondam, Petrarchismo mediato: per una critica della forma antologia, Roma, Bulzoni, 1974, in particolare le pp. 33-36.

${ }^{20}$ La princeps uscì a Valencia nel 1511 e venne ristampata, con aggiunte successive, fino all'ultima edizione di Amberes 1573. Questa opera contiene più di 1200 poesie (alcuni veri e propri poemetti). Cito dalla moderna edizione critica: Hernando Del Castillo, Cancionero general, a cura di Joaquín González Cuenca, Madrid, Editorial Castalia, 2004,V voll.
} 


\section{Movite, lingua, or mai dì'l tuo dolore;}

5. Più volte disposto ho de non te amare.

Riporto la nota del curatore:

La serie de poemas en italiano viene al final de las obras de Tapia, y en las cabeceras de folio se lee que son de Nicolás Núñez, cuyas obras vienen a continuación, introducidas por el epígrafe: «Aquí començan las obras de Nicolás Núńez, y esta primera» (f. 154v). Lo prudente es dejar estos poemas en italiano en el anonimato. Es claramente errónea la denominación de soneto para este poema y los siguientes. En realidad, se trata de tercetos encatenados. Para esta serie de poemas en italiano ha sido sustancial la ayuda de Giuseppe Mazzocchi. No obstante la versión ofrecida sigue siendo provisional. ${ }^{21}$

Nell'anonimato possono anche rimanere il secondo, il quarto e il quinto capitolo, ma almeno il primo deve essere riconosciuto per un testo di Bembo anche se appartiene alle rime rifiutate. ${ }^{22}$

Il capitolo Amar stimol d'amor che hai l'alma acesa è di Gonzaga, presente in 4 manoscritti (attributo al nostro dal solo $\mathbf{F N}_{9}$, gli altri lo presentano adespoto) e mai stampato in Italia, ebbe quindi una circolazione nella penisola iberica ancorché sotto falso nome. ${ }^{23}$

\subsubsection{Le opere che organizzano la produzione poetica}

Solo con gli Elogi historici di alcuni personaggi della famiglia Castiglione $(=\text { Elogi })^{24}$ si incontra un'opera che tenta di ordinare la produzione poetica di Castiglione

${ }^{21} I v i$, vol. IV, p. 196.

${ }^{22}$ Si tratta del testo III delle rime rifiutate dell'edizione Dionisotti e il numero 190 di quella Donnini.

${ }^{23}$ Giacomo Vagni presenta l'edizione interpretativa di questo testo in un suo recente articolo (Giacomo Vagni, "L'«honorata schiera» della duchessa Elisabetta. Ipotesi attributive sul Tirsi di Baldassar Castiglione e Cesare Gonzaga”, «Aevum» LXXXVII (2013), pp. 734-758, alle pp. 752-753), ma sembra non conoscere i testimoni $\mathbf{M i}_{1}$ e $\mathbf{V}_{14}$ (cfr. p. 740n).

${ }^{24}$ La sezione che interessa si trova alle pp. 401-464 (elogio LXX, Il Conte Baldassar Secondo formator del Cortegiano). 
presentandola in maniera unitaria e, pur non trattandosi di una stampa a tal fine composta, merita una certa attenzione. Nelle prime pagine dell'elogio leggiamo una breve biografia che si alterna al ricordo della produzione poetica: la prima notizia in tal senso riguarda l'Alcon, seguita dalla ripubblicazione del sonetto Superbi colli e dalla traduzione in latino dello stesso. La motivazione della ristampa del sonetto è subito chiara: da noi ristampato qui per esser stato datto in luce sotto il nome d'Incerto, nel secondo libro delle rime di diversi Auttori; ${ }^{25}$ curiosa invece la premessa alla versione latina: tradotto anche in latino, per arrichirne quello idioma.

Notizie molto interessanti si leggono nelle pagine seguenti:

Altra volta, altri duo sonetti fece il Conte co i medesimi spiriti del primo [vale a dire Superbi colli], et per aventura per la medesima cagione di un amor troppo alto, e troppo sublime; i quali con un ritratto di bellissima, et principalissima Signora, di mano di Rafael Sanzio da Urbino, pose dietro ad un grande, e bellissimo specchio, che si poteva aprire, et chiudere, da chi sapeva l'artificio; dove scritti di sua mano dell'anno 1517 furono ritrovati del 1560 dalla Contessa Caterina Mandella, che fu poi sua Nuora, nel far rinovar la logora cassa dello specchio, e tergere la luce di quello. Questi come gioie preciosissime, e singolari, tratti da i tesori della poesia toscana; furono participati a Cavaglieri di bello spirito. Ma perché nel nono libro delle rime di diversi $[=\mathbf{1 5 6 0}$ ], furono con errori importantissimi stampati; conviene, che qui corretti si leggano. ${ }^{26}$

Seguono i testi di Quando il tempo e di Ecco la bella fronte.

Nel proseguo della trattazione vengono citati gli altri cinque componimenti apparsi precedentemente a stampa, ma vengono riportati solo i primi due versi di ogni poesia:

per chi desiderasse di sapere quali sono i sui componimenti, porrò i principii soli, poiché furono stampati correttissimi dal Dolce, et dal Ruscelli, Maestri di questa lingua. ${ }^{27}$

\footnotetext{
${ }^{25}$ Elogi, p. 410.

${ }^{26}$ Ivi, pp. 411-412.

${ }^{27}$ Ivi, p. 413.
} 
Appaiono così per la prima volta riuniti insieme tutti gli otto componimenti già pubblicati.

La trattazione che Antonio Beffa Negrini fa delle opere poetiche non si interrompe con l'elenco dei testi già editi nelle antologie ma si dilunga anche sull'egloga Tirsi, a proposito della quale viene ricordata la collaborazione tra Castiglione e Cesare Gonzaga, la stesura per una recita durante un Carnevale, la prima stampa presso Paolo Manuzio e una ristampa uscita finalmente sotto il nome d'incerto in Bologna, col titolo della Dea del Metauro. ${ }^{28}$

A tal proposito, pare importante citare anche questo passo:

Compose altri sonetti, et canzoni, e madrigali, il Conte, in gratia della Sig. Duchessa predetta; i quali, scritti in vago carattere in pergameno, in un libro coperto di velluto nero, con lettera d'istesso Cesare Gonzaga, diede alla Sig. Duchessa; et il Duca Guido Ubaldo secondo, donò esso libro al Conte Christoforo secondogenito del Conte Camillo, mentr'era al suo servigio: dal quale habbiamo noi tratto copia, come facciamo di quanto ci viene alla mano, composto da quel scrittore, le quali poesie sono simili all'altre, delle quali si è datto saggio di sopra. ${ }^{29}$

Purtroppo non vengono fornite ulteriori informazioni né altri testi tratti da questa che, se fosse informazione veritiera, sarebbe una testimonianza dell'esistenza di una raccolta organica di poesie dedicate a Elisabetta Gonzaga, cui collaborò Cesare Gonzaga. Inutile dire che questo manoscritto - o la menzionata copia di esso - non è stato ancora ritrovato, anche se sembra di potervi accostare il testimone $\mathbf{R N}$ : ma per le problematiche riguardanti questo codice si veda la sezione 3.6.

Opere volgari e latine del Conte Baldassar Castiglione (= Opere) del 1733 è la seconda stampa che tenta di sistematizzare la produzione poetica di Castiglione. Questo volume è il primo che presenta tutto il corpus fino ad allora conosciuto delle liriche castiglionesche in volgare e in latino ${ }^{30}$ ed è il primo che a queste

\footnotetext{
${ }^{28}$ Come già Vela, non sono riuscito a rintracciare questa ristampa.

${ }^{29}$ Elogi, p. 415.

${ }^{30}$ La produzione latina non sarà oggetto di trattazione; al riguardo si veda Giovanni Parenti, Per Castiglione latino, in Per Cesare Bozzetti. Studi di letteratura e filologia italiana, a cura di Simone
} 
aggiunge anche il Tirsi in principio della raccolta (traendolo, esplicitamente, dalla princeps aldina). All'egloga segue la già nota canzone Manca il fior giovenil e poi un'altra canzone Sdegnasi il tristo cor, per la prima volta pubblicata:

La Canzone II. si pubblica ora per la prima volta da un Codice MS. cartaceo, contenente Rime di diversi, segnato del numero VI. esistente presso il Chiarissimo Sig. Apostolo Zeno. ${ }^{31}$

Questa stampa è degna di nota anche per il fatto che riporta una serie di varianti nel margine alla canzone Manca il fior giovenil e al sonetto Superbi colli, e ancora di più per il fatto che evidenzia il problema attributivo di Euro gentil: questo componimento viene stampato quale ultimo testo, in carattere corsivo, ed è assegnato dai curatori a Ercole Strozzi (come già in 1551 anche se con incipit diverso: Lascivo Euro).

Un ulteriore arricchimento del corpus di rime avviene nel 1760 quando esce il volume Poesie volgari e latine del Conte Baldassar Castiglione aggiuntevi alcune rime e lettere di Cesare Gonzaga (= Poesie). Sono qui presentate autonomamente tutte le rime di Castiglione (inserendo, quindi, anche la canzone Sdegnasi il tristo cor pubblicata in Opere) al quale viene per la prima volta aggiunta la canzone di Gonzaga D'uno in altro desir nel finale, correttamente attribuita a Cesare. La principale novità consiste nella pubblicazione di un gruppo di sei testi che sono anteposti al corpus delle nove liriche già note. Si tratta, nell'ordine, di 4 sonetti, 1 canzone e 1 ballata (anche se a lungo è stata definita una stanza di canzone), ${ }^{32}$ per la prima volta stampati sotto il nome di Castiglione:

\footnotetext{
Albonico et al., Milano, Fondazione Arnoldo e Alberto Mondadori, 1996, pp. 185-218, Giovanni Parenti, Introduzione, edizione, traduzione e commento a quattro carmina di Baldassar Castiglione, in Per Domenico De Robertis. Studi offerti dagli allievi fiorentini, a cura di Isabella Beccherucchi et al., Firenze, Le Lettere, 2000, pp. 345-397 e Anna Maria Salvadè, Per un carme del Castiglione latino, in Imitar gli antichi. Appunti sul Castiglione, Milano, Unicopoli, 2006, pp. 11-32.

${ }^{31}$ Opere, p. 328.

${ }^{32}$ In effetti si trova la citazione di questo componimento, come canzone, all'interno dell'opera di Guglielmo Gorni, Repertorio metrico della canzone italiana dalla Origini al Cinquecento. (REMCD), Firenze, Franco Cesati, 2008, p. 180; è il metro 14.006, descritto coma stanza di canzone dallo schema AbBA.CDEDCEEfFA, mentre si tratta di una ballata dallo schema XyYX ABCBAC CdDX (identico metro di RVF XI).
} 
1. Se al veder nel mio volto or fiamma ardente

2. Quando fia mai, ch'io vi riveggia ed oda

3. Molti gravi sospir in debil core

4. Amor, s'altro non son ch'esser mi soglia

5. Amor, poiché'l pensier, per cui sovente

6. Mentre fu nel mio cor nascosto il foco

Nelle Annotazioni che seguono i testi delle rime e delle lettere di Gonzaga, e che precedono il testo dei carmi latini, si legge:

Questo sonetto [cioè Se al veder nel mio volto] come li tre altri seguenti, e le due prime Canzoni escono ora per la prima volta alla luce, mercè della generosità e finissimo gusto di Monsignor Luigi Valentini Gonzaga, che mi prestò quel Manoscritto prezioso, che vedesi citato da Antonio Beffa Negrini a car. 414, degli Elogj Istorici d'alcuni Personaggi Illustri della Famiglia Castigliona. ${ }^{33}$

Il sonetto Euro gentil è l'ultimo testo, prima della canzone di Gonzaga, e viene stampato facendo precedere ogni verso da due virgolette, ma nelle note finali non è più attribuito a Ercole Strozzi come accadeva in Opere: rimane un testo di dubbia paternità.

L'ultima stampa che presenta tutti e sedici i testi è Lettere del conte Baldassar Castiglione (= Lettere) curate da Serassi al pari di Poesie. Le uniche novità da segnalare sono pertinenti all'ordine dei testi: apre la silloge il Tirsi, seguito dalle due canzoni, da Mentre fu nel mio cor, dai nove sonetti sicuramente castiglioneschi, da Euro gentil sempre presentato con le virgolette prima d'ogni verso, e infine dalla canzone di Gonzaga.

Dopo questa stampa, e prima degli interventi otto-novecenteschi, abbiamo un solo altro testimone di questo tipo: si tratta di Ariosto Castiglione Fracastoro Sannazaro Casa. Canzonieri del secolo XVI (= Canzonieri), che presenta tutte i testi raccolti in Poesie con lo stesso ordine - manca solo la canzone di Gonzaga — ed Euro gentil, stampato facendo precedere ogni verso da due virgolette.

Per mera curiosità bibliografica segnalo che in occasione delle nozze Castiglioni - Pescasio (giugno 1974) uscì un opuscolo per nozze che raggruppa

\footnotetext{
33 Poesie, p. 113 e sgg.
} 
tutti i componimenti noti di Castiglione (con l'eccezione del Tirsi, non pertinente), presentati nell'ordine di Lettere. ${ }^{34}$

\section{Eliminazione delle stampe descripte}

Collazionando i testi presenti in $\mathbf{1 5 4 5}$ e nelle due ristampe si osserva che nessuna variante o errore è registrabile, se non pochissime discrepanze riguardanti i segni diacritici: resta invariato, nelle seconde due, l'ordine dei testi e l'attribuzione, erronea come si cercherà di dimostrare, a Castiglione di Euro gentil. Per queste ragioni le ristampe non verranno prese in considerazione.

Discorso analogo si deve fare per 1547 e per la ripubblicazione dell'anno seguente: la seconda si dichiara ristampa della prima e si può verificare che la lezione non è in alcun punto modificata e che Superbi colli è anche qui, come in 1547, posto tra le liriche della sezione Incerti. Conseguentemente l'edizione del 1548 non deve essere utilizzata per la ricostruzione del testo che trasmette.

1573 tramanda gli stessi testi presenti in 6-1553, vale a dire il sonetto L'alta catena, Amor e la canzone Manca il fior. La stampa del '73, pur presentando un frontespizio completamente differente rispetto a quella del ' 53 e non avendo la lettera di dedica che si può leggere nella prima, in realtà è una diversa emessione di 6-1553: il solo primo fascicolo è stato ricomposto, ma i successivi sono quelli del '53 ed è sufficiente a dimostrarlo il richiamo Libro VI che si può leggere in calce a c. A1r di entrambe le edizioni.

Per quanto riguarda i testi presenti in Opere e già editi in precedenza, sono le Annotazioni (pp. 328-30) a fornire puntuale indicazione delle fonti. Nello specifico:

1. il Tirsi deriva da Stanze;

2. Cantai, mentre nel cor dalla ristampa del 1549 di 1545;

3. L'alta catena, amor da 6-1553;

4. Superbi colli da Elogi (le varianti in margine derivano da collazione di 1547);

5. Ecco la bella fronte e Quando il tempo da Elogi;

6. Manca il fior da $\mathbf{1 5 5 0}$ (le varianti in margine derivano da collazione di 1553);

\footnotetext{
${ }^{34}$ Rime d'amore di Baldassar Castiglione, Mantova, Grassi, 1974.
} 
7. Euro gentil deriva da $\mathbf{1 5 4 5}$ o da una sua ristampa poiché Serassi scrive: fu tratto dal lib. I. delle Rime di diversi ${ }^{35}$ e questo titolo potrebbe indicare 1545 o una delle due ristampe. Dal momento che Cantai è esplicitamente tratto dall'edizione del 1549 ritengo probabile che anche questo sonetto derivi dalla stessa stampa. In ogni caso il testo della lirica presenta due varianti rispetto all'edizione del 1549 (e naturalmente rispetto a 1545 e alla ristampa del (46): al v. 5 potè di contro a puote e al v. 13 sentia invece di sento e credo che queste siano innovazioni dovute a un intervento editoriale.

Per quanto riguarda i componimenti presenti in Poesie:

1. Sdegnasi il tristo cor, Ecco la bella fronte e Quando il tempo sono sicuramente derivate da Opere e sono le Annotazioni a garantirlo;

2. Manca il fior da $\mathbf{1 5 5 0}$ e le varianti in calce derivano da collazione di 6-1553 per espressa dichiarazione in nota. Occorre segnalare che, di fatto, sia il testo sia la nota sono quelle presenti in Opere: credo molto più probabile che questa canzone derivi da lì (a sua volta Opere derivava da 1550 e 6-1553);

3. Euro gentil da Opere, avendo in comune le varianti sopra ricordate;

4. D'uno in altro desir da $\mathbf{1 5 6 5}$ secondo quanto riportato nelle note; c'è solo una variante, che credo una innovazione di Poesie: nell'ultimo verso la cinquecentina legge per riveder cui non veder fu il meglio, mentre la settecentina per riveder cui non veder fu meglio;

5. per Cantai e Superbi colli non abbiamo una dichiarazione, nelle note, che ci possa informare donde derivino; noto solo che non sono più presenti le varianti in margine a Superbi colli. L'identità di lezione con Opere unita al fatto che gli altri testi derivano dalla stampa cominiana potrebbero far supporre identica trafila. Comunque, non fornendo alcuna variante ed essendo probabilmente descripti, non terrò conto di Poesie in relazione a questi due sonetti.

Lettere è con ogni probabilità descriptus di Poesie: sono dichiaratamente tratti da quest'ultima stampa i sei testi per la prima volta ivi stampati; lo confermano in primis l'errore congiuntivo al v. 2 di Sdegnasi il tristo cor e la variante fu meglio in D'uno in altro desir, inoltre Manca il fior presenta la consueta doppia lezione e, infine, Euro gentil riporta le varianti potè e sentia (che da Opere si sono trasmesse

\footnotetext{
${ }^{35}$ Opere, p. 327.
} 
a Poesie). Benché gli altri testi non presentino varianti, ritengo improbabile che derivino da altre fonti. In ogni caso, Lettere non sarà utilizzato come testimone.

Canzonieri deriva da Lettere: lo dimostrano l'identico ordine e la concordanza in errore in Euro gentil, Sdegnasi il tristo cor e D'uno in altro desir; pertanto questo testimone non sarà usufruito.

Di seguito si riportano gli incipit dei componimenti della vulgata, con indicazione del metro, della princeps e, tra parentesi, l'indicazione dei testimoni portatori di varianti; l'ordine segue quello cronologico della prima sede di stampa: ${ }^{36}$

1. Amar stimol d'amor che hai l'alma accesa, capitolo ternario, $\mathbf{1 5 1 4}^{37}$

2. Queste lagrime mie, questi sospiri, ballata (parte del Tirsi), 1545

3. Cantai mentre nel cor lieto fioria, sonetto, 1545

4. Euro gentil, che gli aurei crespi nodi, sonetto, 1545

5. Superbi colli, e voi sacre ruine, sonetto, 1547

6. Manca il fior giovenil de' miei prim'anni, canzone, 1550 (6-1553)

7. Tirsi, egloga, Stanze

8. L'Alta catena, Amor, la fiamma ardente, sonetto, 6-1553

9. Quando il tempo, che 'l ciel con gli anni gira, sonetto, 1560 (Elogi)

10. Ecco la bella fronte e il dolce nodo, sonetto, 1560 (Elogi)

11. D'uno in alto desir quest'alma stanca, canzone, $\mathbf{1 5 6 5}$

12. Sdegnasi il tristo cor talor, s'avviene, canzone, Opere

13. Se al veder nel mio volto or fiamma ardente, sonetto, Poesie

14. Quando fia mai, ch'io vi riveggia ed oda, sonetto, Poesie

15. Molti gravi sospir in debil core, sonetto, Poesie

16. Amor, s'altro non son ch'esser mi soglia, sonetto, Poesie

17. Amor, poiché 'l pensier, per cui sovente, canzone, Poesie

18. Mentre fu nel mio cor nascosto il foco, ballata, Poesie

\footnotetext{
${ }^{36}$ In questo elenco non si tengono in considerazione gli interventi otto-novecenteschi poiché forniscono sempre l'indicazione del manoscritto dal quale sono cavati i testi e, conseguentemente, non sono utili al fine della ricostruzione critica del testo. Ovviamente, tali contributi, hanno una capitale importanza per la storia della fortuna e degli studi.

${ }^{37}$ Come detto supra, questa ballata uscì nel 1514 musicata da Tromboncino nel volume Frottole libro undecimo che, in quanto stampa musicale, non viene preso in considerazione.
} 


\subsubsection{Le pubblicazioni otto-novecentesche di inediti}

Dopo la pubblicazione dei sei inediti in Poesie, il corpus delle rime dei due cugini consta di 17 componimenti (18 considerando anche Queste lacrime mie che, come è noto, è parte del Tirsi). Non stupisce dunque che contributi anche recenti citino Castiglione sulla base delle stampe del Settecento e dei pochi interventi successivi che hanno portato alla luce testi inediti.

Il primo contributo viene da Cian che nel 1942, studiando un gruppo di carte autografe, ${ }^{38}$ pubblica una serie di testi: ${ }^{39}$

1. una serie di giochi in rima;

2. un abbozzo di sonetto di 9 versi Sotto altro cielo e piu diverso clima;

3. Se per un sguardo o per rider finto (sonetto);

4. Da qual parte del ciel et dal qual choro (sonetto);

5. Scrisse già frate Carlo a un vicino (sonetto).

Un ulteriore e cospicuo accrescimento del corpus poetico si deve a Dionisotti che nella sua recensione alla monografia del maestro cura l'edizione di 5 sonetti: ${ }^{40}$

1. Io penso spesso, se 'l pensar me accora;

2. Quando amor per mia dolce e acerba pena;

3. Cesare mio, qui sono ove il mar bagna;

4. Da poi ch'in puerile e verde etade;

5. Io dico spesso: Amor, che dà tal vena.

\footnotetext{
${ }^{38}$ D'ora in poi nominate Carte mantovane o CM; rimando alla sezione relativa, 3.3, per i problemi attributivi posti dall'insieme dei testi contenuti in questi fogli.

39 Vittorio Cian, "Nel mondo di Baldassarre Castiglione", Archivio Storico Lombardo, VII (1942), pp. 9-97.

${ }^{40}$ Carlo Dionisotti, "Recensione a Vittorio Cian, Un illustre nunzio pontificio", Giornale storico della letteratura italiana, CXXIX (1952), pp. 67-83.
} 
Nel 1977 Stefani ${ }^{41}$ pubblica le sette ottave che componevano un intermezzo della commedia Eutichid ${ }^{42}$ recitata nelle feste urbinati del 1513. Per un inquadramento di questi versi rimando al paragrafo 3.5.

Altri due sonetti sono resi noti da Balduino: ${ }^{43}$

1. A che più a un infelice far ritorno;

2. Ho fondato un castello in ferma sede.

L'ultimo intervento, in ordine di tempo, è la stampa dell'intero corpus poetico (28 poesie e 69 giochi in rima) che si trova nelle Carte mantovane in una monografia dovuta a Michela Fantato. ${ }^{44}$ Come già annunciato, tornerò sui problemi posti da questo «giacimento».

Questo elenco offre la storia maggiormente nota - e spesso citata - degli interventi novecenteschi che hanno aumentato in misura considerevole il corpus poetico di Castiglione. Ma, benché spesso vulgato, quanto scritto non offre un quadro esauriente. Nel monumentale lavoro in 4 volumi di Francesco Trucchi ${ }^{45}$

\footnotetext{
${ }^{41}$ Luigina Stefani, “Le «Ottave d'Italia» del Castiglione e le feste urbinati del 1513", Paragone, CCCXXXII (1977), pp. 67-83.

${ }^{42}$ Il testo delle ottave si può leggere anche in: Nicola Grasso, Eutichia, a cura di Luigina Stefani, Messina-Firenze, D’Anna, 1978.

${ }^{43}$ Armando Balduino, Due sonetti inediti di Baldassar Castiglione, in Ventitré aneddoti raccolti nell'Istituto di Filologia e Letteratura italiana dell'Università di Padova, a cura di Ginetta Auzzas e Manlio Pastore Stocchi, Vicenza, Neri Pozza, 1980, pp. 33-39, ripubblicato in Armando Balduino, Due sonetti inediti di Baldassar Castiglione, in Periferie del petrarchismo, a cura di Beatrice Bartolomeo e Attilio Motta, Roma-Padova, Antenore, 2008, pp. 115-122 in quest'ultima sede, al saggio segue una lettera di Dionisotti a Balduino nella quale si avanzano alcuni dubbi sulla cronologia ipotizzata da Balduino per Ho fondato un castello in ferma sede, ma si conferma l'importanza del ritrovamento dei due sonetti.

${ }^{4}$ Baldassarre Castiglione, Rime e giochi di corte, a cura di Michela Fantato, prefazione di Giancarlo Malacarne e Gilberto Pizzamiglio, Mantova, Universitas mercatorum Mantuæ (volume monografico dell'omonima rivista), 2004.

${ }^{4}$ Poesie italiane inedite di dugento autori dall'origine della lingua infino al secolo decimosettimo raccolte e illustrate da Francesco Trucchi, 4 voll., Prato: Ranieri Guasti, 1846-7 (= 1846-7).
} 
troviamo due sonetti che ci interessano: Amor tu puoi veder di certo homai di Gonzaga ${ }^{46}$ e Io dico spesso, amor, chi dà tal vena di Castiglione. ${ }^{47}$

Questa pubblicazione offre il terzo testo di Cesare accanto alla canzone D'uno in altro desir più volte stampata e al capitolo Amar stimol d'amor. Per quanto riguarda il componimento di Castiglione, devo osservare che si tratta dell'ultimo pubblicato da Dionisotti e questo fatto, senza nulla togliere ai meriti di quel contributo, dimostra solo che di $\mathbf{1 8 4 7 - 8}$ non si è mai tenuto conto in merito alle rime dei due cugini.

\subsection{Tradizione manoscritta}

La fortuna manoscritta delle rime di Castiglione e Gonzaga è abbastanza vasta dal momento che sono 63 i manoscritti che contengono loro testi. Per avere un confronto quantitativo basta segnalare che sono quasi altrettanti i manoscritti latori delle rime di Ariosto, ${ }^{48}$ Bandello ha una diffusione molto minore ${ }^{49}$ mentre, all'estremo opposto, le rime di Bembo sono presenti in circa 200 manoscritti. ${ }^{50}$

Il ms. più cospicuo quanto a numero di testi è $\mathbf{F N}_{3}$ che presenta dieci componimenti: 4 adespoti (una parte del Tirsi, Amaro stimol d'Amor, Sdegnasi il tristo cor e Amor poiché il pensier), 3 attribuiti a Castiglione (Io dico spesso, Cantai mentre nel cor e Da poi ch' in puerile e verde etade) e 3 a Gonzaga (Lasso pur el mio mal, Amor s'altro non son, Lasso quanto fia mai). C'è da dire che l'ultima canzone adespota è situata immediatamente prima della sezione di Baldassarre e che le attribuzioni hanno una forma curiosa: per entrambi gli autori è posta in calce ai singoli testi una abbreviazione del nome; $B a l$. e $C$ e. rispettivamente. Rimando al paragrafo relativo per le problematiche riguardanti l'attribuzione di Amor, s'altro non son.

\footnotetext{
${ }^{46}$ Ivi, vol. III, p. 146.

${ }^{47}$ Ivi, vol. III, p. 198.

${ }^{48}$ Il censimento di Maria Finazzi elenca 68 manoscritti; cfr. Maria Finazzi, Edizione critica delle rime del canzoniere di Ludovico Ariosto, tesi di dott., XVI ciclo, relatore prof. Simone Albonico, Università degli studi di Pavia, 2002-2003.

${ }^{49}$ Il censimento di Danzi rintraccia 22 codici; cfr. Matteo Bandello, Rime, a cura di Massimo Danzi, Modena, Edizioni Panini — Istituto di studi rinascimentali di Ferrara, 1989.

${ }^{50}$ Cfr. Pietro Bembo, Le rime, a cura di Andrea Donnini, 2 voll. Roma, Salerno, 2008.
} 
FN $_{7}$ attesta 3 componimenti di Gonzaga e altrettanti di Castiglione: le liriche di Cesare sono Privi son li occhi miei, So Paleoto mio e Amor tu puoi veder di certo homai; i testi di Baldassarre sono gli stessi presenti ed esplicitamente attribuiti in $\mathbf{F N}_{3}$, con l'inversione del secondo e del terzo.

Infine $\mathbf{F N}_{8}$ presenta una selezione che è possibile trovare in altri codici: Sdegnasi il tristo cor, Cantai mentre nel cor e Queste lagrime mie. Tali testi, nel medesimo ordine, sono presenti anche in $\mathbf{V e}_{3} \mathrm{e}$ in $\mathbf{V} \mathbf{e}_{4}$; si noti inoltre che nel codice fiorentino Euro gentil (attribuito a Strozzi) segue la sezione castiglionesca, mentre in $\mathbf{V e}_{3}$ questo testo è immediatamente prima dei versi di Castiglione. L'analisi delle rubriche dei tre manoscritti potrebbe fornire una ulteriore prova di apparentamento: in $\mathbf{F N}_{8}$ si legge M. Bald. K., in Ve K.K.M. Baldassar da Castion [sic], $\mathbf{V e}_{4}$ invece B.K.M. Balthasar da Castiglion. A proposito della " K" presente in queste rubriche, noto che in molte lettere ${ }^{51}$ autografe Baldassarre rappresentava con una " K» l'iniziale del suo cognome; solo per portare alcuni esempi: lettere 33 e 40 (5 gennaio e 13 febbraio 1505) B.K., 37 e 38 (4 e 9 febbraio 1505) Bal. Kastiglione K. La seconda "K» che si legge in calce alle lettere 37 e 38 (e ad altre) rimanda all'appellativo Charus o Karus che si trova anche nel titolo dell'epistola al re d'Inghilterra (Baldasar Castilionus Charus ad sacratissimum Britannice regem Henricum de Guidobaldo Montefeltrio duce); di questo appellativo sono state fornite due spiegazioni: è da mettere in relazione, secondo Cian, ${ }^{52}$ col recente titolo cavalleresco concesso a Baldassarre prima della sua partenza per l'ambasceria in Inghilterra, ${ }^{53}$ oppure è un riflesso della pretesa di Castiglione di derivare dalla famiglia Caro. ${ }^{54}$ In ogni caso non è per nulla strano trovare due « k» nel nome del nostro, quale che sia la reale motivazione della seconda.

Per tornare ai nostri tre codici e alla varia resa delle loro rubriche, sembra di capire che il capostipite avesse una indicazione fortemente abbreviata tanto

\footnotetext{
${ }^{51}$ Il testo di riferimento dal quale desumo questa informazione e, in generale, estraggo le citazione delle lettere è: Baldassarre Castiglione, Le lettere. 1497-marzo 1521, a cura di Guido La Rocca, Milano, Mondadori, 1978.

${ }^{52}$ Vittorio Cian, Un illustre nunzio pontificio del rinascimento. Baldassar Castiglione, Città del Vaticano, Biblioteca Apostolica Vaticana, 1951, p. 51.

${ }^{53}$ Questa interpretazione è seguita anche da G. La Rocca e la si trova nel commento a tutti i luoghi delle lettere in cui appare.

${ }^{54}$ Baldassarre Castiglione, Vita di Guidobaldo duca di Urbino, a cura di Ubaldo Motta, Roma, Salerno, 2006. L'editore, commentando il passo dell'epistola (pp. 4-5), fornisce bibliografia al riguardo.
} 
da poter essere ricopiata in vario modo, e credo che la forma che si legge in $\mathbf{V e}_{4}$ (B.K.M. Balthasar da Castiglion) lo testimoni: è probabile che il capostipite avesse in intestazione solo una sigla poi sciolta variamente e finanche presentata assieme a uno scioglimento. Faccio notare che la « M» posposta in $\mathbf{V e}_{3}$ e $\mathbf{V e}_{4}$ sarebbe da sciogliere M[antovano], mentre, preposta nel fiorentino, dovrebbe dare M[esser].

Due manoscritti padovani sono parzialmente affini ai tre sopra analizzati: $\mathbf{P d}_{2}$ presenta le tre liriche già menzionate e anche la poesia di Strozzi, ma l'ordine è differente: (Euro gentil è dopo Cantai e Queste lagrime mie è a distanza). Questo codice presenta anche Molti gravi sospir attribuita a Gonzaga. ${ }^{55} \mathbf{P d}_{1}$, invece, presenta isolata e all'inizio Manca il fior e poi, in gruppo compatto, Euro gentil, Cantai e Sdegnasi; seguono a distanza Superbi colli e Cesare mio (quest'ultimo non attestato altrove). ${ }^{56}$

RN è un testimone particolare: si tratta di 4 carte che originariamente formavano una lettera composta da due fogli piegati a metà, contenenti 6 testi, nell'ordine: Se al veder, Quando fia mai, Molti gravi sospir, Amor saltro non son, Mentre fu nel mio cor e Amor poiché 'l pensier. Dato che questo testimone pone molti problemi relativamente alla paternità di alcune delle rime contenute (paternità in dubbio tra Baldassarre e Cesare), rimando alla sezione relativa per la trattazione particolareggiata; aggiungo solo che questo manoscritto sembra essere una testimonianza decisiva dell'esistenza di un canzoniere dedicato alla duchessa di Urbino contenente rime dei due cugini.

Gli altri codici paiono, da un punto di vista complessivo, poco interessanti: degno di menzione è $\mathbf{V}_{7}$ che trasmette $\mathrm{i}$ "sonetti dello specchio" facendoli precedere dalla rubrica Del Conte Baldessar Castiglione trovati in un muro di Casa sua in Mantova dove era un specchio et l'effigie d'una donna; questi sonetti sono i primi due testi dell'intero manoscritto e il Vaticano testimonia dunque la diffusione di due testi contornati da una aura particolare.

Si veda la tabella 1 per la sinossi dei testimoni delle rime di Castiglione, la tabella 2 per quella dei testi di Gonzaga e, infine, la tabella 3 per l'elenco dei testimoni delle rime di altri autori erroneamente attribuite a Castiglione da studi precedenti (per le dimostrazioni relative, rimando infra).

\footnotetext{
${ }^{55}$ Per i problemi attributivi riguardanti questo sonetto rinvio al successivo paragrafo 3.6.

${ }^{56}$ Lultimo sonetto è pubblicato in Dionisotti, "Recensione a Vittorio Cian, Un illustre nunzio pontificio", cit., p. 44.
} 
Per l'edizione critica della rime di Baldassarre Castiglione e Cesare Gonzaga

Tavola 1: Sinossi dei testimoni delle rime di Castiglione

\begin{tabular}{|l|l|}
\hline Testo & Testimoni \\
\hline A che più a un infelice & $\mathbf{F N}_{4}$ \\
\hline Amor, poiché 'l pensier per cui sovente & $\mathbf{F N}_{3}, \mathbf{R N}$, Poesie \\
\hline Cantai mentre nel cor & $\mathbf{B A}_{2}, \mathbf{B U}_{4}, \mathbf{F N}_{3}, \mathbf{F N}_{7}, \mathbf{F N}_{8}, \mathbf{F M}_{1}, \mathbf{F M}_{2}$, \\
$\mathbf{P d}_{1}, \mathbf{P d}_{2}, \mathbf{P v}, \mathbf{V}_{18}, \mathbf{V e}_{3}, \mathbf{V e}_{14}, \mathbf{W r}_{\mathbf{r}}, \mathbf{1 5 4 5}$
\end{tabular}




\begin{tabular}{|l|l|}
\hline Alma ebriacha, che da cerchii sciolta & $C M$ \\
\hline Anima mia gentil, io parto e lasso & $C M$ \\
\hline A quel che per superbia alza la cresta & $C M$ \\
\hline Da qual parte del ciel et da qual choro & $C M$ \\
\hline Scrisse già frate Carlo a un mio vicino & $C M$ \\
\hline Se per un sguardo o per un rider finto & $C M$ \\
\hline Signora, hor che fra noi non si ritrova & $C M$ \\
\hline Signora, il dissi, et hor giurando il dico & $C M$ \\
\hline Signora, i'vo pensando & $C M$ \\
\hline Signora, una gran piaga & $C M$ \\
\hline Spiriti illustri, che purgate i chiostri & $C M$ \\
\hline Voi, che di perle e d'hostro & $C M$ \\
\hline Egloga Tirsi & $\mathbf{F N}_{1}, \mathbf{V}_{9}, \mathbf{F N}_{3}$ (ottave XLIII-LV), Stanze \\
\hline Queste lagrime mie, questi sospiri & $\begin{array}{l}\mathbf{P d}_{2}, \mathbf{F N}_{8}, \mathbf{V e}_{1}, \mathbf{V e}_{3}, \mathbf{V e}_{4}, \mathbf{V}_{5}, \mathbf{1 5 4 5} \\
\mathbf{F N}_{1}, \mathbf{V}_{9} \text { e Stanze nel Tirsi) }\end{array}$ \\
\hline
\end{tabular}

Tavola 2: Sinossi dei testimoni delle rime di Gonzaga

\begin{tabular}{|l|l|}
\hline Testo & Testimoni \\
\hline Amar stimol d'amor m'ha l'alma accesa & $\mathbf{F N}_{9}, \mathbf{M i}_{1}, \mathbf{V}_{14}, \mathbf{F N}_{3}, \mathbf{1 5 1 4}$ \\
\hline Amor, s'altro non son ch'esser mi soglia & $\mathbf{F N}_{3}, \mathbf{R N}$, Poesie \\
\hline Amor tu puoi veder di certo homai & $\mathbf{F N}_{7}$ \\
\hline D'uno in altro desir quest'alma stanca. & $\mathbf{W i}, \mathbf{1 5 6 5}$ \\
\hline Lasso quando fia mai che per mia pace & $\mathbf{B U}_{1}, \mathbf{F N}_{3}$ \\
\hline Lasso pur el mio mal tacer voria & $\mathbf{F N}_{3}$ \\
\hline Mentre fu nel mio cor & $\mathbf{R N}$, Poesie \\
\hline Molti gravi sospir in debol core & $\mathbf{P d}_{2}, \mathbf{V e}_{2}, \mathbf{R N}$, Poesie \\
\hline Privi son gli occhi miei di quella luce & $\mathbf{F N}_{2}, \mathbf{F N}_{7}$ \\
\hline So, Paleoto mio, che 'l nome vostro & $\mathbf{F N}_{7}$ \\
\hline
\end{tabular}


Tavola 3: Sinossi dei testimoni delle rime spurie o dubbie

\begin{tabular}{|c|c|}
\hline Testo & Testimoni \\
\hline \multicolumn{2}{|c|}{ NB: indico con Mad. i Madrigali di Cassola (Venezia, 1544) } \\
\hline $\begin{array}{l}\text { Chi vuol veder opra celeste et rara } \\
\text { (L. Cassola) }\end{array}$ & CM, Mad. \\
\hline $\begin{array}{l}\text { Crin d'oro crespi, inanellati et tersi } \\
\text { (G. Volpe) }\end{array}$ & $\mathbf{U B}, \mathbf{U C}, \mathbf{V}_{8}, \mathbf{1 5 4 5}, C M$ \\
\hline $\begin{array}{l}\text { Euro gentil, che gl'aurei crespi nodi } \\
\text { (E. Strozzi) }\end{array}$ & $\begin{array}{l}\mathbf{B A}, \mathbf{B C}_{2}, \mathbf{F N}_{8}, \mathbf{P d}_{1}, \mathbf{P d}_{2}, \mathbf{V}_{6}, \mathbf{V}_{8}, \mathbf{V e}_{3}, \\
1545,1551\end{array}$ \\
\hline $\begin{array}{l}\text { Fiumme gentil che volgi pure e chiare } \\
\text { (I. Bonfadio) }\end{array}$ & $\mathrm{BC}_{1}, \mathbf{1 5 5 8 , 6 - 1 5 5 1 , C M}$ \\
\hline $\begin{array}{l}\text { Ligiadra donna del mio cor regina } \\
\text { (B. Estense) }\end{array}$ & $\mathbf{V}_{1}$ \\
\hline $\begin{array}{l}\text { Per far il mondo pien di maraviglia } \\
\text { (L. Cassola) }\end{array}$ & $\mathbf{V}_{1}, C M$, Mad. \\
\hline Per far un bello intero (L. Cassola) & CM, Mad. \\
\hline $\begin{array}{l}\text { Poscia che sotto ' Ciel nostro intelletto } \\
\text { (I. Bonfadio) }\end{array}$ & $1558,6-1551, C M$ \\
\hline $\begin{array}{l}\text { Quando nella mia donna io penso et } \\
\text { guardo (L. Cassola) }\end{array}$ & CM, Mad. \\
\hline Quando per farsi honore (L. Cassola) & CM, Mad., Vat. Capp. 74 \\
\hline
\end{tabular}

\section{Questioni attributive}

\subsection{Euro gentil}

Il sonetto Euro gentil è forse il caso più antico di problema attributivo all'interno delle rime di Castiglione: per quanto riguarda la tradizione a stampa, 1545 assegna al Conte il testo mentre $\mathbf{1 5 5 1}$ lo presenta come di Ercole Strozzi (con una variante nell'incipit: Lascivo euro). Elogi, come già ricordato, riporta solo i primi due versi senza soffermarsi troppo su questo sonetto, mentre in Opere il testo, pur presente, è attribuito in nota allo Strozzi. Da qui in poi il sonetto sarà sempre presente nelle 
opere che riportano la produzione poetica di Castiglione, ma sarà stampato in coda ai testi e con le virgolette prima di ciascun verso.

Giacomo Vagni, in un suo articolo del 2011, ${ }^{57}$ risolve la questione: Euro gentil è un testo di Ercole Strozzi. L'autore, oltre a trattare di questo sonetto, fornisce l'edizione critica e commentata del corpus poetico dello Strozzi. ${ }^{58}$

\subsection{Superbi colli}

La più famosa poesia di Castiglione è senza dubbio Superbi colli, ma la fortuna di questo testo si è sempre accompagnata a una incertezza attributiva. Il sonetto è collocato nella sezione degli incerti in $\mathbf{1 5 4 7}$ (la princeps del testo) e in $\mathbf{B a}$; nei manoscritti $\mathbf{B A}_{1}, \mathbf{B U}_{3}, \mathbf{F M}_{2}, \mathbf{M i}_{2}$ e $\mathbf{V}_{3}$ è adespoto; mentre nei seguenti testimoni è assegnato ad altri autori: $\mathbf{B A}_{3}$ (Ariosto), $\mathbf{F M}_{1}$ (L. Martelli), $\mathbf{F N}_{1}$ (Sannazaro, attribuzione poi cassata), $\mathbf{S i}_{1}$ (Giuliano de’ Medici), $\mathbf{V}_{10}$ (F. Brunelleschi). Accanto a questi 12 testimoni che presentano il sonetto adespoto o diversamente attribuito, ci sono 23 codici che lo attribuiscono correttamente al nostro. ${ }^{59}$ La tradizione a stampa, dopo l'intervento di Elogi sopra analizzato (cfr. paragrafo 2.1.1), è quasi unanime in merito alla paternità; l'unica stampa a me nota che toglie il sonetto al Conte è il Tesoro di concetti poetici, ${ }^{60}$ che lo assegna al Guidiccioni.

Tra la fine dell'Ottocento e l'inizio del secolo successivo Alfred Morel-Fatio pubblicò due interventi riguardanti il nostro sonetto ${ }^{61}$ nei quali è messa in dubbio la paternità castiglionesca del testo, ma con argomentazioni tutt'altro che solide.

\footnotetext{
57 Giacomo Vagni, "Su un sonetto di Ercole Strozzi già attribuito a Baldassar Castiglione", Aevum, LXXXV (2011), pp. 751-775.

${ }^{58} \mathrm{Si}$ segnala che il sonetto in questione è presente anche nei manoscritti $\mathbf{B A}_{2}$ e $\mathbf{B C}_{2}$, non usufruiti dall'autore.

${ }^{59}$ Si tratta dei testimoni: $\mathbf{B U}_{2}, \mathbf{F e}, \mathbf{F N}_{5}, \mathbf{F N}_{6}, \mathbf{F N}_{9}, \mathbf{F R}, \mathbf{F o}, \mathbf{L u}, \mathbf{M o}, \mathbf{P d}_{1}, \mathbf{P v}, \mathbf{P c}, \mathbf{R C}, \mathbf{G c}, \mathbf{S i}_{2}$, $\mathbf{T d}, \mathbf{V}_{4}, \mathbf{V}_{6}, \mathbf{V}_{12}, \mathbf{V}_{13}, \mathbf{V}_{15}, \mathbf{v}_{17}$ e $\mathbf{W} \mathbf{r}$.

${ }^{60}$ Tesoro di concetti poetici [...] ridotti sotto capi per ordine d'alfabeto da Giovanni Cisano [...], 2 voll., Venezia: Evangelista Devechino e Giovan Battista Pulciano, 1610; il passo si trova nel vol. I, a p. 825 .

${ }^{61}$ Alfred Morel-Fatio, "Histoire d'un sonnet", Revue d'histoire litteraire de la France, I (1884), pp. 97-101 e Alfred Morel-Fatio, "A propos du sonnet Superbi colli", Bulletin Italien, III (1903), pp. 37-38.
} 
È dimostrabile che Superbi colli uscì dalla penna di Castiglione: accanto alla tradizione analizzata sopra, si può allegare come prova l'autografia di Mo e la firma ivi presente in calce al sonetto.

Già da tempo si è avanzata l'ipotesi che il testo del sonetto contenuto nel ms. estense sia autografo e Francesco Flamini nel 1891, parlando di questo codice, notava:

importano ora a noi alquanti foglietti autografi conservati in esso codice $[\ldots]$ un altro sonetto è sottoscritto $B$. Castilioneus. ${ }^{62}$

Cian, polemizzando con Morel-Fatio, afferma:

Godo, infatti, di poter dare la più sicura dimostrazione dell'autenticità giovandomi d'un documento che più probativo non potrebbe essere, cioè l'autografo d'una redazione anteriore alla definitiva, esistente nella Biblioteca Estense di Modena. Il singolare documento merita d'essere qui riprodotto scrupolosamente di sulla trascrizione eseguitami dalla gentile signorina dott. Fornieri. ${ }^{63}$

Purtroppo Cian non cita la segnatura del ms. in questione e non dimostra l'autografia del testo, come prima di lui non fece Flamini.

Grazie ai documenti sicuramente autografi, e recentemente riprodotti all'interno degli Autografi dei letterati italiani ${ }^{64}$ credo si possa accertare l'autografia castiglionesca di Mo.

Si prendano, ad esempio, le riproduzioni 2 e 6 presenti nel volume citato: si tratta di due lettere, la prima scritta da Castiglione per Elisabetta Gonzaga e indirizzata a Ludovico Bagnolo (datata 27 aprile 1510) ${ }^{65}$ e la seconda indirizzata

\footnotetext{
${ }^{62}$ Francesco Flamini, “Jacopo Corsi e Tebaldeo”, Giornale storico della letteratura italiana, XVIII (1891), pp. 395-396.

${ }^{63}$ Cian, Un illustre nunzio pontificio del rinascimento. Baldassar Castiglione, cit., p. 206 le questioni relative alle «redazioni» del sonetto, evocate da Cian, saranno discusse in sede di edizione del testo.

${ }^{64}$ Roberto Vetrugno, Baldassar Castiglione, in Autografi dei letterati italiani. Il Cinquecento, a cura di Matteo Motolese et al., Roma, Salerno, 2009, pp. 135-147.

${ }^{65}$ Archivio di Stato di Mantova, Archivio Gonzaga 1069, c. 93.
} 
alla madre Aloisa Gonzaga (datata 30 agosto 1524). ${ }^{66}$ Sono molte le particolarità che caratterizzano la scrittura castiglionesca:

- la 'c' maiuscola va sotto il rigo e si completa con un arco che comprende all'interno anche le lettere immediatamente seguenti. Esempi: Canossa (riproduzione 2, r. 3; l'arco comprende la prima 'a' e si congiunge alla seconda asta della 'n'), Contento (r. 5; l'arco comprende le lettere 'o' e la prima 'n'), Cavalli (riproduzione 6, r. 4; l'arco comprende l'intera parola).

- La ' $\mathrm{t}$ ' maiuscola è composta da due tratti: il primo è l'asta superiore che però parte con un ricciolo verso il basso e non si completa oltre l'asta verticale, il risultato è una sorta di 7. Esempi: Tutti (riproduzione 6, rr. 3 e 10), Tutta (r. 4), Tutto (r. 6).

- La 'p' minuscola è composta di due tratti: l'asta verticale che ha un ricciolo in basso e si completa sopra l'occhiello (l'occhiello non è eseguito contestualmente, ma la penna deve alzarsi). Esempi: possibile (riproduzione 2, r. 6), parole (riproduzione 6, r. 2).

- La 'm' minuscola ha di solito le aste (soprattutto la prima) più inclinate rispetto alla 'n'. Si confrontino le parole menar (riproduzione 6, r. 5) e menarò (r. 9) e si noti la maggiore inclinazione delle 'm' rispetto alle 'n'.

- La 'h' minuscola è scritta a partire dall'alto e senza sollevare la penna per tracciare la parte bassa della lettera, di modo che l'asta alta spesso non è completa e non poggia sul rigo. Esempi: $a(n)$ chor (riproduzione 2, r. 9) e chio (riproduzione 6, r. 2).

- Particolarità secondarie:

- la 'e' minuscola ha quasi la forma di una odierna 'c' con un tratto che si innalza sul rigo in corrispondenza dell'occhiello non completamente disegnato;

- la 'l' minuscola non ha occhiello.

Queste caratteristiche si trovano tutte sia nelle riproduzioni citate sopra sia nel testo di Superbi colli trasmesso da Mo; in particolare:

- la 'c' di Colossi (v. 5) ha il consueto arco che comprende le lettere 'o' e 'l';

${ }^{66}$ Forlì, Biblioteca comunale, Raccolte Piancastelli, sez. Autografi secoli XII-XVIII, 14, Castiglione Baldassar. 
- la ' $\mathrm{t}$ ' di Theatri (v. 5) e di Triomphal (v. 6) ha la forma caratteristica di 7;

- tutte 'p' (es: opre v. 5) sono del tipo descritto sopra;

- nelle ' $m$ ' è possibile osservare una inclinazione delle aste maggiore rispetto alle 'n'; si confronti Tormento v. 14;

- le 'h' hanno la consueta forma, ad es. ch'el (v. 2) e $A$ h (v. 3).

Anche se alcune delle particolarità descritte hanno una certa diffusione (la 'e' e la 'h' non forniscono prova decisiva), le altre sono molto caratteristiche (su tutte la 'c' e la ' $t$ ' maiuscola) e denunciano inequivocabilmente la grafia di Mo come castiglionesca.

Ultimo indizio è fornito dalla sottoscrizione presente nell'Estense in basso a destra (B. Castilioneus): nessun altro codice presenta il nome dell'autore in veste latina nonostante le diverse rubriche attestino molte varianti del nome e del cognome, mentre sappiamo che Baldassarre talvolta si firmava, nelle lettere, con la variante latina del cognome (Castiglionensis lettera del 16/11/1497, Castiglionus lettera del 02/04/1501 e 05/10/1501, etc.). ${ }^{67}$ Noto che sono soprattutto le lettere del primo periodo ad avere la firma latina: i primi viaggi a Roma si collocano negli stessi anni, periodo e occasione nei quali probabilmente compose il sonetto.

\subsection{Le Carte mantovane}

Con il termine Carte mantovane identifico quel gruppo di carte autografe che si conservano presso la famiglia Castiglioni e che contengono una serie di testi poetici, accanto ad alcuni testi in prosa. La prima notizia di questo "giacimento" è fornita dal Cian:

In un grande cofano di Casa Castiglioni si annida un fascetto di fogli volanti, disordinati e senz'alcuna numerazione che hanno attirato la mia curiosità $[. .$.$] la maggior parte di essi si manifestano a primo$ tratto come scritti dalla mano di Baldassarre Castiglione [...] i componimenti che ne risultano sono vari di forma e di natura [...] per un gruppo numeroso si ha la certezza trattarsi di quei giuochi in rima $-i$ più, in ternari - che, se non per forma metrica, pel

\footnotetext{
${ }^{67}$ Castiglione, Le lettere. 1497-marzo 1521, cit., p. 3 e p. 15.
} 
contenuto fanno pensare ai Motti del Bembo [...] nello stesso foglio seguono due sonetti non trascurabili. ${ }^{68}$

Cian descrive sommariamente le carte, nota la presenza dei «sonetti dello specchio" Ecco la bella fronte e Quando il tempo e, infine, pubblica 3 sonetti, una serie di Giochi e un abbozzo poetico cercando di collegare i testi alle circostanze e ai luoghi della vita di Castiglione.

Questo contributo, pur citato spesso, non ha modificato nella sostanza il corpus poetico di Castiglione, poiché i testi pubblicati da Cian non vengono ristampati né vi sono approfondimenti sulle Carte mantovane fino alla loro «riscoperta» sessant'anni dopo.

Giancarlo Malacarne scrive:

Rileviamo infatti da un indice a noi cortesemente fornito dalla famiglia Castiglioni, intitolato "Documenti manoscritti e stampe di spettanza e proprietà della famiglia Castiglioni, conservati a parte, fuori dell'Archivio di Casatico", come parte preponderante del contenuto dell'archivio medesimo (che conserva tra l'altro l'abbozzo autografo di 70 pagine del Cortegiano) non sia stata studiata ed indagata in profondità [...] La munificenza della famiglia Castiglioni [...] ha consentito a noi di prendere visione di un materiale documentario di straordinario interesse, permettendoci inoltre di " cavare» dal giacimento alcuni elementi [...] tra questi una raccolta di componimenti poetici che l'indice sopracitato così registra: " 14 - Componimenti poetici vari - fogli 26, v. scheda allegata». La scheda allegata [...] non è coeva alla stesura dei diversi componimenti, ma risale al XX secolo ${ }^{69}$ e si limita alla pedissequa trascrizione o dei titoli delle opere o del loro primo verso [...] solo due [scil. componimenti] sono chiaramente indicati come sonetti di altri poeti. Ciò equivale a dire che la stragrande maggioranza del lavoro parrebbe attribuibile al Castiglione, con molti sonetti autografi. Dopo aver valutato l'importanza del materiale a nostra disposizione, ci siamo

\footnotetext{
${ }^{68}$ Cian, "Nel mondo di Baldassarre Castiglione", cit., pp. 41-53.

69 Cian non menziona questa scheda e mi pare proprio che debba essere stata stilata successivamente o contestualmente alla sua visita.
} 
attivati per verificare se tutto o parte di esso fosse stato in passato pubblicato. Da una prima indagine si osserva come solo uno dei sonetti dei quali stiamo dibattendo, risulti in un'edizione delle opere stampata nel XVIII secolo [si cita Opere], mentre per gli altri non è dato, sino a questo momento, conoscere eventuali pubblicazioni. ${ }^{70}$

Dopo alcune note Malacarne stampa la canzone Alma ebriacha, che da cerchii sciolta e un gioco. Preme ricordare che l'intervento del Cian non viene menzionato, anche se il termine "giacimento» era già stato usato dallo studioso per riferirsi al medesimo gruppo di carte, né Malacarne si avvede che in Opere sono presenti due testi e non uno (si dice che solo Quando il tempo è uscito a stampa, e non Ecco la bella fronte presente invece nella medesima pagina di Opere).

Questi errori sono corretti da Michela Fantato nell'introduzione della monografia del $2004^{71}$ nella quale sono stampati tutti i testi delle Carte mantovane (sia le poesie sia i giochi rimati, anche già noti). In questa opera Fantato traccia una sommaria storia della tradizione a stampa delle rime di Castiglione, ricorda l'intervento di Cian e nella Nota al testo fornisce alcune importanti informazioni:

La grafia castiglionesca è riscontrabile nella maggior parte dei testi. L'autografia ci pare dubbia nei componimenti Com'han potuto le varole ascendere, Hor dove sei mia sola et alma speme e Amor che voi più farmi se mia diva. Nei testi autografi la grafia non è peraltro uniforme. $^{72}$

Il principale limite di questa monografia risiede in un errore metodologico: il fatto che tra le carte private di Castiglione ci siano testi poetici che riportano la sua grafia non significa necessariamente che Baldassarre abbia composto quei versi.

Come osservato da Jean-Jacques Marchand in relazione alle rime di Tebaldeo:

\footnotetext{
${ }^{70}$ Giancarlo Malacarne, "Castiglione inedito. Rinvenuto un giacimento letterario da studiare e pubblicare”, Universitas mercatorum Mantuc, 15 (dicembre 2002), pp. 4-7.

${ }^{71}$ Castiglione, Rime e giochi di corte, cit.; il successivo saggio (Michela Fantato, "Baldassarre Castiglione. Rime e giochi di corte", Humanistica, III/1 (2008), pp. 45-54) non modifica quanto sostenuto nella monografia circa la paternità dei testi.

${ }^{72}$ Castiglione, Rime e giochi di corte, cit., p. LIII.
} 
La princeps deriva a sua volta da una silloge — oggi scomparsa — allestita dal Tebaldeo [...] la raccolta, non ancora pronta per la stampa [...] poteva contenere carte estranee al corpus, come per esempio la trascrizione di componimenti di altri autori. ${ }^{73}$

La prima operazione da compiere è quella di verificare se i testi delle Carte mantovane siano effettivamente di Castiglione e non trascrizioni, di sua mano, di versi di altri autori. Cian, studiando un codice di rime appartenuto a Castiglione, segnalava che:

tre carte di esso, e precisamente la $38 v$ (da un sonetto in fuori), la $39 r$ e $v$ e la $40 r$ e $v$, lasciate in bianco dal copista, furono riempite da Baldassarre Castiglione, certamente (come si può desumere, fra altro, dalla scrittura) nella giovinezza sua [...] il codice, scritto verso la metà del Quattrocento, entrò in casa Castiglione circa la fine di quel secolo. ${ }^{74}$

Nelle carte in questione si leggono, oltre a un abbozzo di canzone, sette testi di Sannazaro, due dei quali risultano adespoti: era già noto, quindi, che Castiglione occasionalmente si faceva copista per passione di testi altrui, non sempre segnalando la paternità dei testi che trascriveva in carte private.

Di seguito l'elenco alfabetico dei componimenti pubblicati da Fantato, con l'esclusione dei Giochi:

1. Alma ebriacha, che da cerchii sciolta

2. Amor che voi più farmi se mia diva

3. Anima mia gentil, io parto e lasso

4. A quel che per superbia alza la cresta

5. Chi vol veder opra celeste et rara

6. Crin d'oro crespi, inanellati et tersi

7. Da qual parte del ciel et da qual choro

\footnotetext{
${ }^{73}$ Jean-Jacques Marchand, Strategia attributiva nella poesia cortigiana: il caso di Tebaldeo. Storia dell'arte, musicologia, letteratura, in L'attribuzione: teoria e pratica, a cura di Ottavio Besomi e Carlo Mancuso, Basel, Birkhäuser, 1994.

${ }^{74}$ Vittorio Cian, "Un codice ignoto di rime volgari appartenuto a B. Castiglione", Giornale storico della letteratura italiana, XXIV-XXV (1899-1900), pp. 297-353, 53-93, p. 300.
} 
8. Ecco la bella fronte, e 'l dolce nodo

9. Fiume gentil che volgi pure, e chiare

10. Hor dove sei mia sola et alma speme

11. Meco a batalia i' ti disfido Amore

12. Per far il mondo pien di maraviglia

13. Per far un bello intiero

14. Poscia che sotto 'l cielo nostro intelletto

15. Quando il tempo che 'l ciel con gli anni gira

16. Quando nella mia dea io penso et ardo

17. Quando per farsi honore

18. Se per un sguardo o per rider finto

19. Scrisse già frate Carlo a un mio vicino

20. Signiora, una gran piaga

21. Signora, hor che fra noi non si ritrova

22. Signora, il dissi, et hor giurando il dico

23. Signora, i' vo pensando

24. Sotto altro cielo e più diverso clima

25. Spiriti illustri che purgate i chiostri

26. Voi che di perle, e d'hostro

Già l'editrice dell' intero corpus pubblica i testi Indarno veggio ben che m'affattico e Com'han potuto le varole ascendere (quest'ultimo di autografia non certa) in appendice, poiché le carte dove sono trascritti riportano come intestazione rispettivamente Sonetto dil Boatto e Sonetto del [...]. Nel primo caso Cian notava che il Boatto in questione dovrebbe essere Antonio Boatto, « oscuro rimatore, padovano, del primo Cinquecento [...] petrarchista non dei peggiori», ${ }^{75}$ ma che questo sonetto non è presente nelle Thevolane, unica opera a stampa del padovano a lui nota. La supposizione di Cian è corretta: a c. 34 de Le nuove rime con l'amoroso concetto del Boatto ${ }^{76}$ si legge infatti il sonetto Indarno veggio ben che miaffatico. ${ }^{77}$

\footnotetext{
${ }^{75}$ Cian, "Nel mondo di Baldassarre Castiglione", cit., p. 52 e n.

${ }^{76}$ Stampa uscita senza indicazioni tipografiche nel 1540. Le altre edizione di Boatto censite da Edit16 sono Lo amoroso concetto, Venezia, s.n., 1538 e L’Angelica del Boatto [...], Venezia, Venturino Ruffinelli, 1542 (presso il medesimo stampatore, nello stesso anno, escono anche le Thevolane).

77 Ringrazio Ilaria Borro per aver gentilmente controllato per me questa stampa presso la Biblioteca Nazionale Braidense di Milano.
} 
Tornando alle altre poesie delle Carte mantovane, sicuramente castiglioneschi sono solo i testi 8 e 15 attestati da stampe e manoscritti: si tratta dei citati sonetti dello specchio. I problemi sorgono con i testi 9 e 14: questi due componimenti non sono inediti, trovandosi entrambi in 6-1553 e 1558 e sempre attribuiti a Iacopo Bonfadio (il primo si trova anche 1563 e nel ms. BC B $_{1}$. Anche alcune importanti antologie moderne, tra le quali i Poeti del Cinquecento, ${ }^{78}$ menzionano questi due testi come di Bonfadio nella nota al testo. L'azione descritta dal testo 9 è ambientata sulle rive di un mare o un lago e di un fiume: al di là della convenzionalità topica, il fatto che Bonfadio fosse di Salò pare una riconferma, tanto più che al v. 10 il testo delle Carte mantovane legge fedel pastor al dipartir del giorno mentre le stampe che attribuiscono il testo a Iacopo riportano Fadio pastor al dipartir del giorno con una evidente firma interna che elimina un aggettivo che leggendo l'intero testo pare non molto pertinente. Per quanto riguarda il testo 14 , invece, il v. 18 nelle Carte mantovane è ipermetro mentre non lo è nelle stampe sopra ricordate (questa vita ch'alcun dice, è quasi un prato I questa vita, alcun dice, è quasi un prato) e anche qui l'ambiente descritto pare rinviare al Garda Queste a me care, et honorate rive / non cosi udran a me nei colli intorno (vv. 25-6).

Rimangono alcuni problemi, uno su tutti quello cronologico. Iacopo Bonfadio nacque nel 1508 e occorre pensare a una precoce, ma non impossibile, vena poetica del futuro storiografo di Genova per spiegare il fatto che Castiglione potè copiare un suo testo al più tardi nel 1527 . Una spiegazione alternativa sarebbe quella di negare che la trascrizione sia di mano di Castiglione, ma risultando impossibile consultare le Carte mantovane non si può che affidarsi alle conclusioni dei precedenti editori circa la grafia dei testi.

Il testo 6 apparve a stampa attribuito a Girolamo Volpe in $\mathbf{1 5 4 5}$ (e nelle stampe derivate) ed è presente adespoto in $\mathbf{V}_{8}$ e in $\mathbf{U B}$ (e nel descriptus UC). Purtroppo Girolamo Volpe è poco più che un nome dato che le moderne storie della letteratura non lo citano e il DBI non è utile al riguardo. Le uniche notizie si devono alle storie letterarie del '700: il Crescimbeni fornisce scarne informazioni, ${ }^{79}$

\footnotetext{
${ }^{78}$ Guglielmo Gorni et al. (a cura di), Poeti del Cinquecento. Poeti lirici burleschi satirici e didascalici, Milano-Napoli, Ricciardi, 2001, p. 450.

${ }^{79}$ Cfr. Giovanni Mario Crescimbeni, Istoria della volgar poesia [...], Roma, Chracas, 1698, p. 435: Girolamo Volpe Comasco. Vedi della Patria ciò, che si dice, ove si parla di Gio. Antonio Volpe. Girolamo ha Rime nel lib. I di Diversi eccellentiss. Autori, e in altre raccolte, e fu in credito di buon Rimatore. Ho consultato la ristampa corretta, riformata e notabilmente ampliata del 1714 . Nelle
} 
il Quadrio non aggiunge nulla, anzi deriva dal Crescimbeni, ${ }^{80}$ mentre dà qualche notizia in più la Storia di Como di Maurizio Monti:

Fratello di Giovanni Antonio è Girolamo Volpi. Questi scrisse una vasta opera di geografia, e la intitolò a Carlo Quinto. Ebbe fama a' suoi tempi di principe de' cosmografi; ma credesi che la sua opera non sia stata impressa, e non so se ne esiste copia manoscritta. Sono stampati nella citata Cominiana ${ }^{81}$ quattro suoi epigrammi; altri dodici componimenti poetici sono in un codice manoscritto di casa Giovio, e tutti spirano greca fragranza. ${ }^{82}$

Altri tentativi di reperire informazioni su Volpe non hanno sortito effetti: il Tiraboschi non fornisce alcuna informazione, come neppure l'opera di Giovan Battista Giovio sui letterati comaschi. ${ }^{83}$

In ogni caso credo che l'attribuzione a Girolamo Volpe sia da ritenere veritiera poiché pare difficile che un testo di un personaggio noto come Castiglione venga attribuito a un rimatore quasi sconosciuto, secondo quanto notato da Franca Brambilla Ageno: ${ }^{84}$

Fra l'attribuzione a uno scrittore famoso, e quella a uno scrittore meno noto, ha ovviamente più probabilità di essere vera la seconda.

stampe miscellanee del XVI sec. si leggono almeno 4 suoi componimenti: Aure soavi che pel cielo sereno, Quai pensieri sono si vili e si negletti, Quel raro stil che di invidia accende e il sonetto in questione. Il primo testo si legge in $\mathbf{1 5 4 7}$ (e nella ristampa del 1548) e nel ms. $\mathbf{B U}_{2}$ adespoto, mentre gli altri tre in $\mathbf{1 5 4 5}$ (e nei derivati).

${ }^{80}$ M. Girolamo Volpe, Comasco. Fu egli in credito di buon Rimatore (Francesco Saverio Quadrio, Della storia e della ragione d'ogni poesia, 4 voll., Bologna, F. Pisarri, 1739-1749, vol. II, p. 352) ${ }^{81}$ Joannis Antonii Vulpii Carminum libri quinque. Accesserunt Joannis Antonii Vulpii antiquioris patricii et episcopi Novocomensis, ac Hieronymi ejus fratris carmina que supersunt, Padova, Giuseppe Comino, 1742.

${ }^{82}$ Storia di Como scritta da Maurizio Monti professore nel liceo diocesano della stessa città, 2 voll. Como, Pietro Ostinelli, 1829-1832, vol. II, pp. 697-8.

${ }^{83}$ Giovan Battista Giovio, Gli uomini della comasca diocesi nelle arti, e nelle lettere illustri, Bologna, Forni, 1975, ristampa anastatica dell'edizione Modena, Società Tipografica, 1784.

${ }^{84}$ Franca Brambilla Ageno, L'edizione critica dei testi volgari, Padova, Antenore, 1984, p. 219. 
Questo criterio è, nelle questioni di autenticità, il corrispettivo del criterio della lectio difficilior nella scelta delle lezioni. ${ }^{85}$

Di conseguenza è opportuno attribuire a Volpe questo sonetto.

I testi 2 e 11 riportano in calce, rispettivamente, Fr.P. e C.Mor.: queste due indicazioni paiono essere delle rubriche attributive le quali, però, non mi risultano trasparenti poiché non riesco a scioglierne le abbreviazioni. I due testi in esame, allo stato attuale delle ricerche, non sembrano attestati altrove e in ragione di ciò, e in considerazione del fatto che il sonetto 2 pare avere una grafia non sicuramente castiglionesca, ritengo prudente considerarli di dubbia paternità.

Per quanto riguarda gli altri testi, anche $5,12,13,16,17$ non sono inediti, in quanto apparvero nei Madrigali di Luigi Cassola stampati a Venezia nel $1544 \mathrm{e}$ ristampati l'anno successivo. ${ }^{86} \mathrm{Si}$ tratta di una opera molto particolare trattandosi di una raccolta (e non di una stampa musicale) composta esclusivamente da madrigali, sostanzialmente un unicum nella nostra storia letteraria. ${ }^{87}$

Oltre ai Madrigali del 1544, si trovano 25 poesie di Cassola nelle antologie a stampa del cinquecento ${ }^{88}$ e ci sono moltissime occorrenze di suoi madrigali in stampe musicali. Il piacentino è sicuramente il più importante madrigalista del primo cinquecento.

\footnotetext{
${ }^{85}$ Sulla «forza di attrazione» che il nome di un autore famoso esercita nei confronti di testi di autori meno noti si veda: Alfredo Stussi, Introduzione agli studi di filologia italiana, Bologna, il Mulino, 2003, p. 112 e Emanuela Scarpa, Per l'edizione di un poeta cinquecentesco: sulle rime di Giovanni Muzzarelli, in La critica del testo. Atti del convegno di Lecce, 22-26 ottobre 1984, Roma, Salerno, 1985, pp. 531-560, p. 543 e sgg.

${ }^{86}$ Madrigali del magnifico signor cauallier Luigi Cassola piacentino, Venezia, Gabriel Giolito, 1544; la ristampa del 1545 uscì con analogo frontespizio e presso il medesimo editore.

${ }^{87}$ Cfr. Cfr. Niccolò Poggiali, Memorie per la storia letteraria di Piacenza, 2 voll., Piacenza, Niccolò Orcesi, 1789, pp. 161-9; Claudio Vela, Luigi Cassola e il madrigale cinquecentesco, in Tre studi sulla poesia per musica, Pavia, Aurora edizioni, 1984, pp. 29-65; Giuliano Bellorini, "Luigi Cassola madrigalista", Aevum, LXIX (1995), pp. 593-615, Luigi Cassola, Il canzoniere del codice Vaticano Capponiano 74, a cura di Giuliano Bellorini, Piacenza, Tip.Le.Co., 2002 e Giuliano Bellorini, Il magnifico Signor Cavallier Luigi Cassola Piacentino. Edizione critica dei madrigali. Censimento e indice dei capoversi di tutte le rime, Firenze, Olschki, 2012.

${ }^{88}$ Due madrigali e due sonetti in $\mathbf{1 5 4 5}$ (ma nelle ristampe non troveremo più questi testi), un madrigale in 1551 e 15 madrigali 4 sonetti e 1 ballata in 1560. Di questi 25 componimenti solo 2 sono presenti in Madrigali e 7 sono comuni al canzoniere manoscritto.
} 
Se è vero che la fortuna a stampa delle sue opere è abbastanza vasta, non altrettanto si può dire di quella manoscritta: a parte una isolata presenza in $\mathbf{B} \mathbf{C}_{2}$ e il gruppo di madrigali di Wr. ${ }^{89}$ è il Capponiano 74 della Biblioteca Apostolica Vaticana a conservarci un intero canzoniere del piacentino. Questo manoscritto contiene un progetto di canzoniere organico (e non una somma di componimenti come invece è il volume di Madrigali) anche strutturalmente bembesco: 110 sonetti, 8 canzoni, 21 madrigali, 4 sestine, 2 ballate e una ode. Alcune spie, ad esempio le indicazioni del tipo «non si ponga», fanno supporre il progetto di una stampa, poi non avvenuta; complessivamente la stesura del canzoniere è stata datata da Vela agli anni 1543-44.

Dopo queste brevi notazioni ritorniamo ai testi delle Carte mantovane: 5, $12,13,16,17$ sono presenti nei Madrigali di Cassola e 16 è attestato anche dal canzoniere Capponiano. Saremmo in presenza, quindi, di cinque madrigali composti da Cassola e trascritti da Castiglione nelle sue carte private.

Il confronto tra la lezione di alcuni componimenti rafforza questa ipotesi (riporto nelle colonna di sinistra il testo tradito dalle Carte mantovane e in quella di destra la trascrizione dei Madrigali di Cassola).

\section{Esempio 1}

Chi vol veder opra celeste e rara vegga questa mia dea et sua beltade
Chi vuol veder opra celeste, et rara vegga questa mia donna, et sua beltade

Il v. 2 del componimento 5 nelle carte di Castiglione potrebbe risultare corretto solo a patto di leggere come bisillaba dea, ma questa parola in Bembo è sempre un monosillabo..$^{90}$ Osservando il Tirsi si nota che la parola dea è presente

\footnotetext{
${ }^{89} \mathrm{Al}$ riguardo, si veda la successiva sezione.

${ }^{90}$ Bembo, Le rime, cit., 111 v. 1 Già donna, or dea, nel cui verginal; Stanze I v. 8 a la cortese Dea che nel mar nacque, IV v. 2 onoran la lor Dea con pura fede, XIV v. 8 udir tra voi, la Dea strana mi scusi. In Petrarca ci sono 3 occorrenze del termine e si tratta sempre di parola bisillaba ma esclusivamente in sede di rima, quindi non pertinente per il caso in esame; cfr. RVF CLIX v. 5 Qual ninfa in fonti, in selve mai qual dea; CCCXXXVII v. 8 il mio signor sedersi e la mia dea; CCCLXVI v. 98 Or tu, Donna del ciel, tu nostra Dea. Si potrebbe allargare lo sguardo anche ai Trionfi, ma il risultato non cambierebbe: Triumphus Mortis I v. 124 Vattene in pace, o vera mortal dea!
} 
nove volte ${ }^{91}$ ed è sempre un monosillabo. La lezione dei Madrigali risulta, quindi, corretta e la lezione di mano di Castiglione sembra contenere un errore di copia.

\section{Esempio 2}

Quando nella mia dea io penso et ardo amor io giurerei che quanto è bel tra voi, quel tanto è in lei sola se stessa et nulla altra somiglia et veggio in un suo sguardo mille volti, la morte et la mia v[ita] in fin ciò ch'è in costei è maraviglia et è la gracia tanto infinita ch'ardisco dir, e il dico, al mio dispe[tto] ch'il ciel privo di lei non è perfetto
Quando nella mia donna io penso e guardo amor io giurarei che quanto è bel tra voi quel tanto è in lei, sola se stessa, et nulla altra somiglia et veggo in un suo sguardo mille volte la morte e la vita in fin ciò, che è in costei è maraviglia et è la gratia sua tanto infinita ch'ardisco dir, e il dico al mio dispetto ch'il ciel privo di lei non è perfetto

Il primo verso ${ }^{92}$ nelle carte di Castiglione contiene, a mio parere, un errore: ardo, pur essendo plausibile, al confronto con guardo appare una corruzione poiché tutta l'azione descritta nel componimento riguarda l'azione della vista (somiglia, veggio, sguardo, etc.). Il v. 8 del componimento presente nelle Carte mantovane è ipometro a meno di non ipotizzare una improbabile dialefe tra le ultime due parole, mentre nei Madrigali si legge correttamente et è la gratia sua tanto infinita. In questo caso è palese che si tratti di un errore: il possessivo è caduto nell'atto di copia.

\section{Esempio 3}
Quando per farsi honore
Quando per farsi honore

... ...

[v. 16] alor fra noi mostra il bel sembiante vostro

[v. 16] hor fra noi mostra il bel sembiante vostro

\footnotetext{
${ }^{91}$ XII, v. 4 La luna, che è su in ciel si bella dea; XIX, v. 6 Ove è la dea la qual tanto si noma; XXVI, v. 1 Ma se la nostra dea veder vorai; XXXIV, v. 2 Va questa dea cum le sue nymphe errando; XXXV, v. 6 Che a canto de la dea sempre si vede; XXXVI, v. 6 Stassi la dea che tutte l'altre avanza; XXXIX, v. 1 A questa nostra dea tutti i pastori; XLV v. 6 La nostra dea cum le sue nymphe ascolta; XLVI v. 2 Vive la dea che tu cercando vai.

${ }^{92}$ Anche qui abbiamo una variante dea $>$ donna, ma il verso non risulta ipometro poiché tra dea e io si può avere una dialefe.
} 
Il verso 16 nelle Carte mantovane è ipermetro anche se la Fantato nota che esiste una variante sul rigo che riporta qui. Tale correzione è però un tentativo per così dire solamente ortopedico: il madrigale descrive cronologicamente la Creazione (prima mostra... poi ci scopre... et poi) che culmina con l'hor dei Madrigali. Anche in questo caso la lezione di mano di Castiglione pare avere un errore di copia.

Prima di cercare di proporre soluzioni si riportano alcune osservazioni di Vela:

Qui importa notare come nell'ambiente dei musicisti i testi di Cassola avessero una loro notorietà già nel quarto decennio del secolo, molto prima della pubblicazione dei Madrigali. La fama di Cassola come autore di poesie madrigalesche doveva essere assicurata, a Piacenza [...] e fuori Piacenza [...]. Comunque anche le fonti musicali non permettono di spostare i testi di Cassola troppo prima del 1530. Per ragioni che si vedranno, sono i musicisti del nuovo stile madrigalistico a trovare congeniale alle loro musiche i madrigali cassoliani, e d'altra parte lo stile e il numero stesso di questi testi presuppongono esperienze poetiche e musicali difficilmente ipotizzabili prima del $1525-30 .^{93}$

Quanto riportato (e la dimostrazione che, nell'articolo, ne segue) è un elemento che non semplifica la soluzione del quesito. Una data relativamente bassa (1525-1530) per la composizione dei madrigali cassoliani confligge con la presenza dei 5 testi nelle Carte mantovane che, pur non avendo una datazione univoca e certa, sembrerebbero contenere scritti del secondo decennio del secolo (ricordo che le due date presenti in questo fascio di fogli sono il 1513 e il 1519).

Pur in presenza di alcuni elementi problematici, ritengo che i cinque testi siano tutti da attribuire a Cassola. Infatti le ipometrie e l'ipermetria nei componimenti 5, 16 e 17 - presenti nelle Carte mantovane - paiono errori di trascrizione (modifica di donna >dea, caduta di un pronome e banalizzazione hor $>$ alor); Cassola è il compositore di madrigali per eccellenza della prima metà del XVI sec. (mentre non c'è giunto alcun madrigale sicuramente castiglionesco); il fatto poi che due

\footnotetext{
${ }^{93}$ Vela, Luigi Cassola e il madrigale cinquecentesco, cit., p. 43.
} 
di questi cinque testi ${ }^{94}$ siano stati musicati da compositori piacentini (Claudio Veggio su tutti) è un elemento non trascurabile; infine uno di questi madrigali è presente nel canzoniere autografo mai giunto alle stampe e questo fatto credo renda la paternità di quel testo incontrovertibile.

Se questo è vero occorre però retrodatare di almeno un lustro, o al limite di un decennio, l'inizio dell'attività poetica di Cassola e questo fatto non incontra particolari ostacoli.

Per riassumere, quindi, il testo 6 è di Girolamo Volpe; 9 e 14 sono di Iacopo Bonfadio; 5, 12, 13, 16, 17 sono di Luigi Cassola; dei testi 2 e 10 l'autografia è dubbia; i testi 2 e 11 sembrano riportare in calce una attribuzione ancorché abbreviata e non trasparente. Data l'impossibilità di consultare le Carte mantovane è necessario affidarsi alle analisi di Cian e della Fantato, sia per quanto riguarda la lezione dei testi sia per quanto concerne l'autografia.

Prudenzialmente, in un futura edizione critica si dovrebbero pubblicare i testi $1,3,4,7,8,15,18-26$ tra le rime di Castiglione, ipotizzando siano sicuramente castiglioneschi $i$ testi non altrove attestati poiché è ragionevole che una produzione estemporanea e non rifinita rimanga autografa e non abbia circolazione, mentre saranno da pubblicare tra le rime dubbie i testi 2, 10 e 11.

Da quanto è emerso sembra di dover riconsiderare, almeno in parte, le Carte mantovane. Risulta che quasi un terzo dei componimenti non è stato composto da Baldassarre e quindi l'insieme di quelle carte non offre un esempio di libro privato sul quale Castiglione fermò sue poesie, alcune rifinite (si pensi ai sonetti dello specchio) e altre allo stato di abbozzi; si tratta invece di una testimonianza diversa. L'insieme di queste carte testimonia una prassi castiglionesca: abbozzi poetici, madrigali d'occasione, sonetti rifiniti, trascrizioni di altri poeti potevano coesistere all'interno di uno stesso perimetro, senza particolari demarcazioni. Avendo tolto a Baldassarre 7 testi poetici ritenuti suoi, credo però di aver ampliato la conoscenza su come lavorava quando componeva poesia; in ogni caso occorre inserire i nomi di Volpe, Bonfadio, Boatto e Cassola tra i poeti noti a Castiglione.

Infine, ancora una nota sulla monografia di Fantato: a parte le errate attribuzioni che questo contributo ha innescato, anche sul piano ecdotico ci sono alcune imprecisioni, la più importante delle quali riguarda Quando il tempo che ll ciel. Segnalo questo fatto perché il testo, tratto dall'edizione Fantato, sta avendo una

\footnotetext{
${ }^{94}$ Si tratta dei testi 5 e 12.
} 
certa diffusione. ${ }^{95} \mathrm{Nel}$ testo del sonetto, al v. 5 l'editore pubblica questa lezione verran quei, dove ancor qui vita non spira e in nota segnala ch'anchor aggiunto in interlinea (nelle Carte mantovane ci sono due versioni di questo sonetto). La lezione accolta a testo produce però una ipermetria evidente e, in mancanza di una visione dell'autografo, ipotizzo che la variante d'autore in interlinea opponga dove ancor vita non spira (lezione analoga a quella di tutti gli altri testimoni) a ch'anchor qui vita non spira.

\subsection{I madrigali di Wr}

Il manoscritto Wr contiene due testi sicuramente castiglioneschi: Superbi colli a c. $8 \mathrm{r}$, correttamente attribuito, e Cantai mentre nel cor lieto fioria a c. $12 \mathrm{v}$ attribuito a Pietro Bembo. Il manoscritto in questione non è molto autorevole quanto alle attribuzioni dei testi dato che le rubriche sono chiaramente inserite dopo il momento della copiatura dei componimenti (è la collocazione delle stesse a garantire ciò) e in molti casi il testimone incorre in errori (ad esempio dichiara di Bembo 5 poesie non sue e, contemporaneamente, assegna a Vittoria Colonna un testo del cardinale). ${ }^{96}$ Lo stesso testimone sembra attribuire a Castiglione sette madrigali presenti alle carte $143 \mathrm{v}-144 \mathrm{v}$ dei quali, per comodità, riporto gli incipit e la rubrica:

I. c. $143 v$ Da quei begli occhi che pensar mi fanno, Castiglione

II. c. 143v Madonna egli è gran cosa e più che grande, Cas.

III. c. $143 \mathrm{v}$ Dolor della mia doglia, Cas.

IV. c. 144r Vana speranza in cui sperar solia, Cas.

V. c. $144 \mathrm{r}$ Se la durezza in voi fosse men dura, Cas.

VI. c. 144 r Amor poi che Madonna, Cas.

VII. c. $144 v$ Madonna se volete Cas.

I testi I, III, IV, V e VI sono presenti anche nei Madrigali di Cassola, mentre il madrigale II sembra non avere altra attestazione a stampa o manoscritta e un madrigale con identico incipit del VII si può leggere in alcune stampe musicali

\footnotetext{
${ }^{95}$ Il testo dei «due sonetti dello specchio» presenti e pregevolmente commentati in Lina Bolzoni, Poesia e ritratto nel rinascimento, Roma-Bari, Laterza, 2008 è tratto dall'edizione Fantato e di conseguenza ne condivide l'errore.

${ }^{96}$ Cfr. Bembo, Le rime, cit., pp. 652-653.
} 
e nel manoscritto Chigi E V 174 della Biblioteca Apostolica Vaticana a c. 108r attribuito a Luigi Groto.

Come detto sopra, Wr non è fededegno quanto alle attribuzioni: l'inserimento tardivo delle rubriche e la tendenza ad attribuire i testi ad autori noti mina la credibilità di questo testimone. Tornando alle rubriche di questi sette madrigali, si nota che sei di queste hanno la forma Cas. e solo la prima Castiglione; credo che in queste tracce - e nel comportamento complessivo del copista - ci possa essere la spiegazione: probabilmente l'antigrafo di Wr riportava il gruppo di madrigali attribuiti a Cassola con una rubrica quale Cas. e il copista del nostro manoscritto avrà tentato uno scioglimento del compendio attribuendo il gruppo a un autore per lui familiare (a c. 8, ricordo, c'è un testo di Castiglione, mentre non ci sono testi esplicitamente attribuiti a Cassola).

Non ci sono ragioni per attribuire i testi II e VII a Castiglione in quanto essi o sono pezzi estravaganti di Cassola o sono madrigali di attribuzione dubbia tra il piacentino e altri madrigalisti del Cinquecento.

\subsection{Le «Ottave d'Italia»}

Con il termine «Ottave d'Italia» ci si riferisce alle 7 ottave che si leggono alle cc. $194 \mathrm{v}-195 \mathrm{v}$ di $\mathbf{V}_{16}$ e costituiscono l'intermezzo, recitato in prosopopea dall'Italia, della commedia Eutichia messa in scena nelle feste urbinati del 1513. Questi versi sono stati a più riprese attribuiti a Castiglione, e da ultimo pubblicati da Luigina Stefani. ${ }^{97}$

L'ultima editrice del testo svolge una convincente argomentazione in favore della paternità castiglionesca delle "Ottave» a partire dalla lettera di Baldassarre a Ludovico Canossa databile tra il 13 e il 21 febbraio $1513 .{ }^{98}$

Stefani, infine, fornisce alcune prove interne accostando il testo delle ottave con quello di Superbi colli. È possibile rintracciare in almeno un altro testo di Castiglione una costellazione lessicale analoga a quella impiegata in una ottava: ${ }^{9}$

\footnotetext{
${ }^{97}$ Stefani, "Le «Ottave d'Italia» del Castiglione e le feste urbinati del 1513", cit.; si rimanda al saggio anche per l'elenco dei precedenti studiosi che hanno attribuito le ottave al Castiglione, pur senza fornire dimostrazioni al riguardo.

${ }^{98}$ Cfr. Castiglione, Le lettere. 1497-marzo 1521, cit., pp. 343-8.

${ }^{99}$ Questo collegamento non è presentato da Stefani.
} 
Ottava IV, vv. 4-8

Barbare genti m'hanno lacerata
Cesare mio, vv. 5-8

Qui la vittoria espetta et Franza e Spagna di sue rapine, e prede mal divise; e chi al barbaro giogo si sommise hor tardo de'l suo error si pente e lagna.

È possibile trovare puntuali riscontri non solo tra le ottave e le altre poesie di Castiglione, ma anche nelle lettere e nel Cortegiano. Già l'editrice delle ottave fa notare alcune corrispondenze con parti del trattato: ${ }^{100}$ libro II $₫$ XXVI ché ormai non resta nazione che di noi non abbia fatto preda, tanto che poco più gli resta che predare e pur ancor di predare non si resta e libro I $\$$ XXXVI invidioso morso del tempo. Occorre dire, però, che se il primo esempio è calzante, il secondo non è di grande aiuto, poiché il passo si riferisce a considerazioni sulla storia della lingua e inoltre è motivo topico.

Tuttavia, seguendo questa strada, è possibile trovare un altro punto di contatto dato che nella prima redazione del proemio, poi non accolta nelle fasi successive, si legge:

Veggiamo tante nobilissime città distrutte, Troia, Sparta, Micene, Athene, e molt'altre che già fur vili hora essere florentissime. Roma, che già fu regina del mondo [Dil mondo fui regina, ottava III v. 1] per virtù de l'arme, e temuta fin negli estremi confini della terra da tante barbare e straniere nationi, hora sol si nomina per la religione, ed è habitata da gente alienissima da l'arme. ${ }^{101}$

Oltre al preciso richiamo segnalato, in tutto il passo riecheggia l'ottava III, ovviamente sostituendo a Roma l'Italia.

In considerazione delle motivazioni esterne e dei sondaggi interni è possibile senźaltro confermare la paternità castiglionesca delle "Ottave d'Italia», le quali vanno inserite a pieno titolo tra le sue rime.

\footnotetext{
${ }^{100}$ Cito da Baldassarre Castiglione, La seconda redazione del «Cortegiano» di Baldassar Castiglione, a cura di Ghino Ghinassi, Firenze, Sansoni, 1968.

${ }^{101}$ Cito da Uberto Motta, Castiglione e il mito di Urbino. Studi sulla elaborazione del "Cortegiano", Milano, Vita e Pensiero, 2003. L'estratto è a p. 38 e riporta le cc. 7r-12v del Vat. Lat. 8204.
} 


\subsection{Baldassarre o Cesare?}

I risultati del censimento dei testimoni di alcune poesie tradizionalmente ${ }^{102}$ attribuite a Castiglione riserva qualche sorpresa.

RN presenta 4 carte molto interessanti (cc. 70-73) che meritano di essere analizzate: a c. $70 \mathrm{r}$ si legge Del conte Baldassar Castiglione cavati da un libro manoscritto di pergamena con la coperta di veluto negro, segue il testo di Se al veder nel mio volto e di Quando fia mai, ch'io vi riveggia et oda (con la rubrica Del med(esi) mo); poi a c. 70v Molti gravi sospiri senza alcuna titolazione e di seguito (con la rubrica Del med(esi)mo) Amor, s'altro non son; a c. 71r Mentre fu nel mio cor (con la rubrica Del med(esi)mo); a c. 72r-73v Amor, poiché 'l pensier con l'intitolazione Canzone del Conte Baldassar Castiglione. A c. 71v si legge M. Alessandro Car(issi) mo, vi mando per l'apportattor d(e)lla p(res)e(n)te doi para de polastri et un paro d'occhi, quali farete. Come indicato da Cian ${ }^{103}$ si tratta di una lettera (i segni di piegatura delle cc. sono evidenti) spedita ad un tale Alessandro contenente una serie di testi poetici. Così Cian:

a quanto sembra [doi para de polastri et un paro d'occhi significa] un paio d'oche, che ancor oggi, nella parlata della zona veneto-lombarda, diventano maschi (oco), come in un accrescitivo toscano, del Pulci (ocone). Si tratta di quattro sonetti, corrispondenti ai quattro primi dell'edizione [...] Serassi [...] cui seguono le canzoni [...] che sono rispettivamente la seconda e la prima nella citata edizione. Si noti che nelle carte del codice romano in testa al secondo sonetto e negli altri componimenti si legge: Del med. (del medesimo Castiglione) [...] l'ignoto cinquecentista del cod. romano che aveva spedito i quattro sonetti e le due canzoni al suo amico Alessandro, insieme con i quattro polli e col resto, li aveva trascritti da quel medesimo libro membranaceo " coperto di velluto nero» dal quale il Beffa Negrini aveva tratto copia. ${ }^{104}$

\footnotetext{
102 Dico tradizionalmente poiché le nuove acquisizioni di testi castiglioneschi, derivanti dalle stampe del ' 700 , sono state accettate senza essere mai vagliate.

${ }^{103}$ Cian, "Nel mondo di Baldassarre Castiglione", cit., p. $21 \mathrm{n}$. ${ }^{104} \mathrm{Ivi}$, p. 21.
} 
Come sottolineato in precedenza, e differentemente da quanto sostiene Cian, della rubrica non c'è traccia in corrispondenza di Molti gravi sospir. Ritengo poi che il maestro di Dionisotti non si avveda di quello che potrebbe essere un gioco sotteso alla lettera: l'amico di Alessandro probabilmente non manda a quest'ultimo del pollame e 6 testi poetici, ma i soli componimenti allegati alla lettera, definendo ironicamente i 4 sonetti doi para de polastri e i 2 componimenti di metro differente un paro d'occhi. ${ }^{105}$ In ogni caso questa attestazione manoscritta ${ }^{106}$ sembra confermare l'esistenza del «canzoniere» citato da Beffa Negrini e fornisce l'antigrafo di Poesie: in quell'edizione, come sopra ricordato, relativamente ai 6 componimenti allora per la prima volta stampati, si notava:

escono ora per la prima volta alla luce, mercé della generosità e finissimo gusto di Monsignor Luigi Valenti Gonzaga, ${ }^{107}$ che mi prestò quel Manoscritto prezioso, che vedesi citato da Antonio Beffa Negrini a car. 414, degli Elogj Istorici d'alcuni Personaggi Illustri della Famiglia Castigliona. ${ }^{108}$

È altamente probabile che l'editore di Poesie non abbia visto il ms. di velluto, ma le carte ora in $\mathbf{R N}$ che si dichiarano esemplate da quel codice, che rimane tutt'ora un fantasma: tutti e soli i testi presenti in $\mathbf{R N}$ vengono stampati in Poesie, e la collazione tra i due testimoni conferma che il primo è l'antigrafo del secondo. ${ }^{109}$

I problemi sorgono poiché la tradizione manoscritta di Molti gravi sospir non si limita al ms. gesuitico, ma comprende anche $\mathbf{V e}_{2}$ e $\mathbf{P d}_{2}$. Questi due codici attribuiscono il testo a Cesare Gonzaga e fanno riferimento a una tradizione

\footnotetext{
${ }^{105}$ Non si tratta di due canzoni, come sostiene Cian, ma di una canzone e di una ballata.

${ }^{106}$ Cian data così le carte in questione: 4 carte di mano dei primi decenni del sec. XVI, anzi direi, sincrone al Castiglione (Cian, "Nel mondo di

Baldassarre Castiglione", cit., p. 21).

${ }^{107}$ Ricordo che RN proviene dalla biblioteca di Valenti Gonzaga e comprende molti testi suoi $\mathrm{o}$ indirizzati a lui o che lo riguardano.

${ }^{108}$ Poesie, p. 113.

${ }^{109}$ Per un interessante commento ai testi presenti in $\mathbf{R N}$ e per uno studio sul contesto della poesia alla corte di Urbino nei primi anni del Cinquecento, rimando a Giacomo Vagni, Una breve raccolta di liriche dedicata ad Elisabetta Gonzaga da Baldassar Castiglione e Cesare Gonzaga, in I Gonzaga e i papi. Roma e le corti padane fra Umanesimo e Rinascimento (1418-1620), a cura di Renata Salvarani, Citta del Vaticano, Libreria Editrice Vaticana, 2014.
} 
diversa del sonetto ma, prima di risolvere i problemi editoriali, rimane il problema attributivo: a rigore Molti gravi sospir è adespoto in $\mathbf{R N}$ e attribuito a C. Gonzaga da $\mathbf{V e}_{2}$ e $\mathbf{P d}_{2}$.

Ma non è tutto: Amor, śaltro non son è attestato da $\mathbf{F N}_{3}$ e questo manoscritto lo attribuisce a Gonzaga. Il codice fiorentino è, assieme a $\mathbf{F N}_{7}$, il più importante collettore di rime di Gonzaga; questi, accanto a $\mathbf{V e}_{2}$ e $\mathbf{P d}_{2}$, sono gli altri due testimoni che attribuiscono esplicitamente liriche a Cesare (le altre attestazioni manoscritte di sue poesie sono sempre adespote). Di più: $\mathbf{F N}_{3}$ presenta adespota, ma appena prima della sezione contenente rime attribuite a Castiglione, Amor, poiché il pensier.

Rimane da capire di chi siano Amor, s'altro non son e Molti gravi sospir. A questo punto è lecito avanzare una ipotesi: il libro di velluto nero, almeno stando alle notizie di Beffa Negrini, contiene testi di Castiglione $i$ quali, scritti in vago carattere in pergameno, in un libro coperto di velluto nero, con lettera d'istesso Cesare Gonzaga, [Castiglione] diede alla Sig. Duchessa. ${ }^{110}$ Pare trattarsi del secondo omaggio poetico che Castiglione fece a Elisabetta, dopo il Tirsi, e, si badi bene, in entrambi i casi il cugino dovette esemplare il manoscritto di dedica. ${ }^{11}$ Ma se per il Tirsi la collaborazione nella scrittura poetica tra i due autori è sicura, non vedo problemi particolari nel ritenere che anche il manoscritto di velluto nero potesse contenere liriche di entrambi. Perché mai Cesare avrebbe dovuto firmare una lettera di dedica di un libro che conteneva esclusivamente poesie di Baldassarre? Ben si capisce che il Beffa Negrini, impegnato a tratteggiare l'elogio del Conte, abbia voluto enfatizzare la parte di Castiglione mettendo in secondo piano la parte di Gonzaga relativamente al codice in questione.

Questa sembra poter essere la soluzione: Molti gravi sospir e Amor, s'altro non son sono poesie di Gonzaga, contenute accanto a liriche di Castiglione nel ms. in lode di Elisabetta. Ricordo che per il primo testo, quanto ad attribuzione, $\mathbf{R N}$ tace mentre $\mathbf{V e}_{2}$ e $\mathbf{P d}_{2}$ (appartenenti a una diversa famiglia) lo assegnano a Cesare; al secondo sonetto $\mathbf{R N}$ antepone Del medesimo, ma il testo immediatamente precedente è proprio Molti gravi sospir, che $\mathbf{F N}_{3}$ dice essere di Gonzaga $\left(\mathbf{F N}_{3}\right.$ rafforza la mia tesi presentando anche Amor, poiché il pensier nel limitare della sezione di Castiglione).

\footnotetext{
${ }^{110}$ Elogi, p. 415.

${ }^{111}$ Ricordo che $\mathbf{V}_{9}$, testo di dedica dell'egloga, è autografo di Gonzaga, con una sua lettera introduttiva.
} 
Se tutto questo è corretto, o plausibile, anche l'attribuzione di Mentre fu nel mio cor, che in RN segue Amor, s'altro non son con la sola indicazione Del medesimo e non ha altre attestazioni manoscritte, vacilla: anche questo testo dovrebbe spettare a Gonzaga e fino a contraria prova lo considero di Cesare.

Mi pare che una riprova significativa stia proprio nelle rubriche di $\mathbf{R N}$ : dopo i tre testi in esame, come detto sopra, è presente la canzone Amor, poiché 'l pensier con l'intitolazione Canzone del Conte Baldassar Castiglione. Perché mai il copista avrebbe dovuto ripetere il nome dell'autore - dopo averlo scritto in testa al primo componimento - se tutti i 4 fogli spediti all'amico contengono rime di Baldassarre? Dopo un testo chiaramente attribuito, uno adespoto e due con l'indicazione Del medesimo, l'ultimo testo (contiguo) dovrebbe avrebbe la stessa rubrica dei precedenti qualora fossero tutti testi di un solo autore, ma la nota in testa alla canzone, di per sé, è segnale che le liriche non sono di un'unica persona.

Le ipotesi non possono confermare le dimostrazioni, ma possono chiarire la fenomenologia di questi fatti. Se davanti a Molti gravi sospir ci fosse l'indicazione Di Cesare G., allora tutto sarebbe chiaro: $\mathbf{R N}$ ci trasmetterebbe, come credo faccia, un testo di Baldassarre, tre del cugino e infine una canzone del Conte. Ritengo sia possibile che il sodale di Alessandro abbia tralasciato quella rubrica o per banale dimenticanza o perché non riusciva a leggere il nome presente nel suo antigrafo. ${ }^{112}$

La tesi opposta, vale a dire ritenere tutti i testi di Castiglione, esige una spiegazione molto meno economica: $\mathbf{V e}_{2}$ e $\mathbf{P} \mathbf{d}_{2}$ da una parte e $\mathbf{F N}_{3}$ dall'altra avrebbero innovato separatamente, modificando l'attribuzione, in maniera identica, togliendo due testi a un autore noto per darli a un molto meno noto (si sa che, normalmente, avviene il contrario). Ipotesi molto meno economica, e perciò fallace: i tre testi sono da attribuire pertanto a Gonzaga.

Si segnala infine che in un recente contributo Giacomo Vagni ${ }^{113}$ attribuisce a Cesare Gonzaga il sonetto Vo fugendo d'amor li occulti inganni e la canzone Perché di lachrimar son stanco homai sulla base di indizi e sondaggi esterni. Nello specifico, i vv. 10-11 della canzone sono presenti nell'ottava 44 del Tirsi e nell'egloga

\footnotetext{
112 Segnalo che la rubrica di Ve 2 legge: C. Gon. e, se il lettore non conosce Cesare Gonzaga, è improbabile che questa abbreviazione gli risulti chiara. Posso allegare una prova: nelle carte manoscritte che conservano il tentativo di descrizione della classe IX dei manoscritti Marciani fatto da Pietro Zorzanello, in corrispondenza di $\mathbf{V e}_{2}$, il grande bibliotecario legge C. Gon[zaga], senza sciogliere il nome proprio e mettendo un punto interrogativo.

${ }^{113}$ Giacomo Vagni, "L’honorata schiera» della duchessa Elisabetta. Ipotesi attributive sul Tirsi di Baldassar Castiglione e Cesare Gonzaga”, cit., pp. 734-758.
} 
viene detto che l'autore del testo è un giovinetto; la canzone in questione si legge adespota alle cc. $144 \mathrm{r}-147 \mathrm{r} \mathbf{F N}_{3}$ subito dopo il sonetto Vo fugendo, il quale a sua volta segue il capitolo Amar stimol d'amor, anch'esso adesposto ma sicuramente di Gonzaga sulla base di $\mathbf{F N}_{9}$. Oltre alla contiguità in uno dei manoscritti che li tramandano con testi gonzaghiani, insomma, non sembrano esserci prove positive che riconducano i due componimenti a Cesare; Vagni, peraltro, è costretto a interpretare in senso traslato l'attributo giovane col quale nel Tirsi si descrive l'autore della canzone, dal momento che Gonzaga aveva 33 anni al momento della rappresentazione dell'egloga.

Nello stessso contributo, Vagni attribuisce il capitolo Dolce e amaro destin, che mi sospinse a Castiglione. Anche in questo caso si tratta di un testo evocato all'interno del Tirsi, all'ottava 41 e attribuito a un poeta mantovano. Il capitolo si può leggere in due mss. e due stampe antiche, ${ }^{114}$ ma nessun testimone lo attribuisce a Castiglione. In attesa di nuove indagini, pertanto, ritengo che questi tre testi siano da considerare quanto meno di dubbia paternità.

\subsection{Lasso quando fia mai}

A parte i testi sopra menzionati in dubbio tra Baldassarre e Cesare, un altro sonetto presenta alcuni problemi attributivi: Lasso quando fia mai, attribuito da $\mathbf{F N}_{3}$ (ricordo: uno dei collettori delle rime di Gonzaga) a Cesare e da $\mathbf{B U}_{1}$ a Bembo e, per questa ragione, troviamo il sonetto come primo delle Rime dubbie nell'edizione Donnini. Come già sostenuto da Vagni, ${ }^{115}$ il manoscritto bolognese presenta molti errori nelle rubriche attributive e, come rilevato da Donnini, ${ }^{116}$ ha la spiccata tendenza a far figurare come del Bembo numerosi testi non suoi. Anche Danzi ${ }^{177}$ rileva che un testo di Bandello (I'volli donna, già contarvi a pieno) è attribuito in maniera errata dal Bolognese a «il Silvano». Credo che questi dati

\footnotetext{
${ }^{114}$ Si veda l'elenco in Giacomo Vagni, "L'«honorata schiera» della duchessa Elisabetta. Ipotesi attributive sul Tirsi di Baldassar Castiglione e Cesare Gonzaga”, cit., p. 756.

${ }^{115}$ Giacomo Vagni, "L'«honorata schiera» della duchessa Elisabetta. Ipotesi attributive sul Tirsi di Baldassar Castiglione e Cesare Gonzaga”, cit., p. 756n.

${ }^{116}$ Due testi di Trissino (Quando ripenso meco al sommo bene, e Il lampeggiar de' begl'occhi sereni), un testo di Niccolò Delfino (L'alte bellezze et la vertù perfecta) sono attribuiti a Bembo. Informazioni desunte da: Bembo, Le rime, cit., pp. 562-564.

${ }^{117}$ Bandello, Rime, cit., pp. 321-322.
} 
garantiscano che $\mathbf{B} \mathbf{U}_{1}$ non è per nulla affidabile quanto ad attribuzioni e, minata l'assegnazione a Bembo del nostro sonetto, rimane solo la rubrica di $\mathbf{F N}_{3}$ che attribuisce il testo a Cesare. Per queste ragioni ritengo che Lasso quando fia mai possa essere definitivamente attribuito a Cesare Gonzaga.

\section{Saggio di edizione}

I testi sono trascritti con criteri conservativi; in sintesi:

1. distinguo secondo l'uso moderno $u$ e $v$;

2. riduco a $i$ la semiconsonante $j$;

3. normalizzo le iniziali maiuscole: le conservo solo in caso di nomi propri e le elimino all'inizio del verso;

4. divido e unisco con moderazione le parole secondo la prassi moderna: non intervengo dove la riunione di parole comporti il raddoppiamento;

5. la congiunzione sarà et davanti a vocale e solo in quel caso;

6. sciolgo le più comuni abbreviazioni senza segnalarlo, in caso di titulus erroneo non lo indico;

7. mantengo l'h etimologica e i nessi latineggianti -ti-, -ph-, -bs-, -pt-, -ct-, -mn-, -nst-, -nsf-;

8. aggiorno e introduco i diacritici: accenti, apostrofi, punteggiatura.

Le innovazioni sopra riportate non saranno segnalate negli apparati critici. Nella fascia di apparato non segnalo le difformità grafico-formali minime presenti nei testimoni.

\subsection{Superbi colli}

Testimoni manoscritti: Mo c. 92r, B. Castilioneus (autografo); Ba p. 185, De incerti autori; $\mathbf{B A}_{1}$ p. 187, adespoto; $\mathbf{B A}_{3}$ cc. 19v-20r, attr. a L. Ariosto; $\mathbf{B U}_{2}$ c. $144 \mathrm{r} ; \mathbf{B U}_{3}$ c. 136r, adespoto; Fe p. 51, Castiglion Baldasare; $\mathbf{F M}_{1}$ c. 134v-135r, Ludovico Martelli; $\mathbf{F M}_{2}$ c. 135 r, adespoto; $\mathbf{F N}_{1}$ c. 52 v, con attribuzione a Sannazaro poi cassata; $\mathbf{F N}_{5}$ c. $109 v$, B. Castiglion; $\mathbf{F N}_{6}$ c. 35r, Di ms Baldasse Castilione; $\mathbf{F N}_{9}$ cc. 26v-27r; FR c. 250v, Baldassarre Castiglione; Fo c. 70r, Del Barignano; Lu p. 335, Baldassarre Castiglione; $\mathbf{M i}_{2}$ c. 130v; Pd 1 c. 30r, di messer Baldesar da Castiglione; Pv p. 70, Baldassar Castiglione milanese; Pc p. 239, di ms. Baldassar da Castiglione; RC c. 17r; 
Gc c. $180 v-181$ r, con attribuzione a Sannazaro poi cassata; $\mathbf{S i}_{1}$ c. $16 \mathrm{r}-\mathrm{v}, \mathrm{IU} M E(=$ Giuliano de' Medici); $\mathbf{S i}_{2}$ c. 52, del conte Baldassarre Castiglio [sic]; Td c. 37r, del Castiglione $; \mathbf{V}_{3}$ c. $84 \mathrm{v}$, adespoto; $\mathbf{V}_{4}$ c. $160 \mathrm{v} ; \mathbf{V}_{6}$ c. $447 \mathrm{r}$, adespoto; $\mathbf{V}_{10}$ c. $46 \mathrm{r}$, attr. a Filippo Brunelleschi. $\mathbf{V}_{12}$ c. $322 \mathrm{r}$, adespoto; $\mathbf{V}_{13}$ c. $91 \mathrm{r}$, incerto; $\mathbf{V}_{15}$ c. $42 \mathrm{v}$, Annibale Caro; $\mathbf{V}_{17}$ p. 157 , Castiglione; Wr c. 8r, Baldessara Castiglione. Testimoni a stampa: 1547 c. 137 r, incerti; 1548 c. 132r, incerti; Elogi p. 410; Opere p. 326.

Sonetto di schema ABBA ABBA CDC DCD.

L'edizione del testo si può avvalere dell'autografo conservato da Mo. Come già ricordato (si veda il pragrafo 2.1.2), Elogi presenta una lezione divergente rispetto all'autografo, e a partire da Opere queste varianti vennero per così dire valorizzate presentando a testo la lezione di Elogi e in margine le due varianti, tratte probabilmente da $\mathbf{1 5 4 7 .}$

Queste sono le varianti che riguardano i versi 5 e 11 (a sinistra la lezione di Mo, che coincide qui con la princeps, a destra quella di Elogi):

theatri, archi, colossi, opre divine

colossi, archi, teatri, opre divine ...

et l'opre e i nomi insieme il tempo atterra et l'opre, e i nomi il tempo invido atterra

Si potrebbe trattare, quindi, di due varianti d'autore come già ha ritenuto Cian:

giovandomi d'un documento che più probativo non potrebbe essere, cioè l'autografo d'una redazione anteriore alla definitiva, esistente nella Biblioteca Estense di Modena. ${ }^{118}$

Cian assume dunque, senza fornire la dimostrazione, che Mo sia la prima redazione ed Elogi la seconda. Se però si analizza tutta la tradizione, il quadro sembra essere più sfumato poiché i testimoni si dividono non in due gruppi come ci si aspetterebbe (vale a dire un gruppo testimoniante la «redazione anteriore» e uno quella " definitiva», per usare la definizione di Cian), ma in quattro.

I manoscritti latori di una lezione analoga a quella di Mo, limitatamente ai vv. 5 e 11, sono i seguenti: Ba, $\mathbf{B A}_{3}, \mathbf{B U}_{2}, \mathbf{F e}, \mathbf{F M}_{1}, \mathbf{F N}_{1}, \mathbf{F N}_{6}, \mathbf{F N}$, FR, Fo, $\mathbf{P d}_{1}$, Pc, RC, Gc, $\mathbf{S i}_{2}, \mathbf{T d}, \mathbf{V}_{3}, \mathbf{V}_{6}, \mathbf{V}_{12}, \mathbf{V}_{15}, \mathbf{V}_{17}$ (gruppo A). I codici che tramandano l'altra lezione sono: $\mathbf{B A}_{1}, \mathbf{L u}, \mathbf{M i}_{2}, \mathbf{P v}$ (gruppo B).

\footnotetext{
${ }^{118}$ Cian, Un illustre nunzio pontificio del rinascimento. Baldassar Castiglione, cit., p. 206n.
} 
Abbiamo però altri due gruppi di testimoni: $\mathbf{S i}_{1}, \mathbf{F N}_{5}$ e $\mathbf{W r}$ (gruppo C) riportano colossi, archi, teatri, opre divine come Elogi ma et l'opre e i nomi insieme il tempo atterra come $\mathbf{M o}$; mentre $\mathbf{B U}_{3}$ e $\mathbf{V}_{13}$ (gruppo D) attestano Archi, theatri, colossi, opre divine (verso ipermetro) e, al v. 11, et l'opre e $i$ nomi insieme il tempo atterra.

I manoscritti del gruppo B sono testimoni tardi, non anteriori al XVIII secolo; non si hanno quindi, allo stato attuale delle ricerche, manoscritti riportanti la supposta variante d'autore del v. 11 testimoniata da Elogi che siano antecedenti a questa stampa.

Ritengo che la variante al v. 11, presente a partire da Elogi, possa non essere d'autore poiché, tra le due, pare facilior quella attestata dal gruppo B, dal momento che attesta una lezione (il tempo invido) fin troppo topica. ${ }^{119}$ Si può inoltre notare che tutte le traduzioni del sonetto postulano, come lezione di partenza et l'opre e $i$ nomi insieme il tempo atterra e non la concorrente: Nec modo res ipsae taciturnis passibus aevi, / ipsa, sed et rerum nomina trita iacent; ${ }^{120}$ iccirco si tempus edax opera omnia solvit / aetas si rabido singula dente rapit; ${ }^{121}$ et bien qu'au temps pour un temps facent guerre / les bastimens, si est-ce que le temps / oeuvres et noms finablement atterre; ${ }^{122}$ y assi puesto que al tiempo hizistes guerra / todo lo acaba el curso $y$ movimiento / del aligero tiempo quando cierra. ${ }^{123}$

Volendo ammettere che la variante della stampa sia d'autore credo si debba invertire la cronologia ipotizzata da Cian: la prima redazione sarebbe quella attestata da Elogi, modificata poi da un intervento d'autore che avrebbe voluto eliminare un luogo molto vulgato. ${ }^{124}$

\footnotetext{
${ }^{119}$ Orazio, Odi, lib. I, 11, vv. 7-8.

${ }^{120}$ Si tratta dei vv. 12-14 della traduzione di Giovanni Flamingo il Giovane (inc.: Endomiae colles Urbis, sacraeque ruinae) presente in Elogi, pp. 410-411.

${ }^{121}$ Vv. 9-10 della versione di Niccolò d'Arco (inc.: Excelsi colles Urbis, sacraeque ruinae), cfr. Opere, p. 329.

${ }^{122}$ Vv. 7-9 della traduzione di Joachim Du Bellay stampata nel 1558 (inc.: Sacrez costaux, et vous sainctes ruines); segnalazione in Morel-Fatio, "Histoire d'un sonnet", cit., p. 97.

${ }^{123}$ Versione di Andrès Rey de Artiera, vv. 9-11, apparsa a stampa nel 1605; la versione spagnola di Gutierre de Cetina, pubblicata nel 1585, non è utile per questo confronto dal poiché si tratta di una riscrittura (notizie in ivi, pp. 100-101]).

${ }^{124}$ Ad analoghe conclusioni giunge (basandosi però sulle sole stampe) Michel Bastiaensen che in maniera sintetica, attenendosi solo alle stampe, giunge a considerazioni analoghe alle
} 
Per quanto riguarda le varianti al v. 5 è possibile eliminare i manoscritti del gruppo B poiché probabilmente derivati da Elogi e i testimoni del gruppo D in quanto latori di lezione errata. Rimangono due varianti, vale a dire theatri, archi, colossi, opre divine (gruppo A), colossi, archi, teatri, opre divine (Elogi più il gruppo C). Si nota che entrambe divergono solo per l'ordine col quale sono presentati i quattro simboli della grandezza di Roma in decadimento. Anche in questo caso sembra più probabile che si tratti di semplici scambi intervenuti in sede di copia e non di varianti imputabili a Castiglione. La lezione di Mo presenta una climax che nella variante concorrente scompare e questo fatto rafforza la tesi che la variante del gruppo C e di Elogi sia il frutto di una corruzione.

Poiché ci è giunto l'autografo di questo testo e poiché mi sembra che nella tradizione si possano osservare solo errori o varianti imputabili ai copisti e ai curatori delle stampe, pubblico il sonetto sulla base di Mo, senza segnalare in apparato le varianti e gli errori degli altri testimoni.

Mantengo le due grafie ipercorrette relliquie del v. 3 e darrà del v. 14 .

Superbi colli, e voi sacre ruine ch'il nome sol di Roma ancor tenete, ah, che relliquie miserande havete, di tant'anime excelse e pellegrine!

Theatri, archi, colossi, opre divine, trïomphal pompe, glorïose e liete, in poche cener pur converse sete, e fatt' al volgo vil favol' al fine.

Così, se ben un tempo al tempo guerra fanno l'opre famose, a passo lento, e l'opre et i nomi insieme il tempo atterra, vivrò dunque fra' miei martir contento, ché s'il tempo dà fine ad ciò ch'è in terra, darrà forse ancor fine al mio tormento.

mie. Cfr. Michel Bastiaensen, Noterella su un verso del Castiglione, presente sul portale online La repubblica letteraria, consultabile all'indirizzo http://www.repubblicaletteraria.it/ BaldassarreCastiglione.html. 


\subsection{Io dico spesso: Amor, chi dà tal vena}

Testimoni: BI c. 92r (acefalo causa incendio), $\mathbf{F N}_{3}$ c. 215r, Bal.; $\mathbf{F N}_{5}$ c. 109r, Bal. Castiglion; $\mathbf{F N}_{7}$ c. 110r, Sonetti di ms. Bald. Castiglioni.

Sonetto di schema ABBA ABBA CDC DCD.

Questo è uno dei sonetti pubblicati da Dionisotti il quale lo trasse da $\mathbf{F N}_{7}$ ma non si accorse che tale sonetto non era inedito, in quanto uscì in 1846-7 con questa rubrica: Estratto dal testo 1192 stroziano $\left(=\mathbf{F N}_{5}\right)$ e ricorretto sul codice 724 magliabechiano $\left(=\mathbf{F N}_{7}\right)$.

Sono presenti due errori che possono dare indicazioni dei rapporti che intercorrono tra i testimoni: al v. $1 \mathbf{F N}_{7}$ legge Io dico spesso amor che dà tal vena, mentre $\mathbf{F N}_{3}$ e $\mathbf{F N}_{5}$ chi dà tal vena (BI non è d'aiuto: il primo verso e parte del secondo non sono leggibili causa incendio). La variante di $\mathbf{F N}_{7}$ è teoricamente ammissibile, ma se scorriamo tutto il sonetto leggiamo chi tanto gli alti miei desiri affrena (v. 5), chi dà vigore (v. 7); la risposta a questa serie di domande è al v. 11: la dolce nostra et di pietà nemica. Con ciò la lezione di $\mathbf{F N}_{7}$ risulta erronea e ritengo l'errore probabilmente separativo, anche in ragione del fatto che Dionisotti, il quale conosceva solo questo manoscritto quale testimone del sonetto, non propose di emendare il luogo in questione.

Al v. 9 abbiamo una situazione speculare ma opposta: $\mathbf{F N}_{7}$ legge ei mi risponde allhor: se ti nutrica; $\mathbf{F N}_{3}$ e $\mathbf{B I}$ se'l ti nutrica, mentre $\mathbf{F N}_{5}$ riporta se'l ti nutrica con la -l- cassata. L'errore risiede in $\mathbf{F N}_{3}$ e $\mathbf{B I}$ e credo che il copista di $\mathbf{F N}_{5}$ abbia copiato fedelmente il suo apografo, accorgendosi poi dell'errore e cancellando la lettera. Non credo che l'errore occorso nei due testimoni (nei tre testimoni prima della correzione di $\mathbf{F N}_{5}$ ) possa essere poligenetico: la loro lezione, oltre a non dare senso, complica la sintassi del verso. Con questo abbiamo un errore congiuntivo e questa corruttela ha una forza dimostrativa maggiore rispetto al precedente. Anche se l'errore al v. 1 non fosse da considerarsi separativo, nondimeno l'ipotesi stemmatica non subirebbe modifiche.

$\mathbf{F N}_{5}$ e BI si oppongono, banalizzando, a $\mathbf{F N}_{3}$ e $\mathbf{F F N}_{7}$ in cinque luoghi: al v. 13 descritta di mia man senza ch'io dica di contro a senza ch'io 'l dica; al v. 12 in questo sol contro a in questi pur e in questo caso c'è ancora una banalizzazione (questi è riferito a occhi suoi di due versi prima) e una ripetizione (sol è presente al v. 10); al v. 6 et dopo chi di contro a et chi dapoi e infine al v. $4 \mathbf{F N}_{5}$ e $\mathbf{B I}$ leggono in si lunghi sospir sì lunga pena, gli altri manoscritti riportano e lunga pena $\left(\mathbf{F N}_{7}\right.$ che sta, stemmaticamente, per conto proprio riporta $e$ scritta sopra al $s i$ cancellato). 
Ritengo corretta la lezione e lunga pena, poiché con la concorrente avremmo un endecasillabo con accenti di 2a, 3a, 6a, 7a, 8a, 10a; la modifica della congiunzione con l'avverbio si può spiegare tramite una ripetizione. $\mathbf{F N}_{5}$ e $\mathbf{B I}$ sono concordi in banalizzazione in cinque luoghi: nessuno dei casi, singolarmente, è monogenetico però ritengo improbabile che due manoscritti che banalizzano cinque volte concordemente siano tra loro indipendenti. Anche se la somma di indizi non fa una prova, ritengo statisticamente probabile che $\mathbf{F N}_{5}$ e $\mathbf{B I}$ siano derivati da un antecedente comune che presentava quei cinque luoghi trivializzati.

Riassumendo: $\mathbf{F N}_{7}$ è probabilmente separato dagli altri testimoni a causa dell'errore al v. 1 mentre $\mathbf{F N}_{3}, \mathbf{F N}_{5}$ e $\mathbf{B I}$ sono congiunti dall'errore al v. 9; $\mathbf{F N}_{5}$ e BI risultano uniti dai cinque luoghi dove banalizzano concordemente. Se l'analisi è corretta, lo stemma codicum dovrebbe essere il seguente:

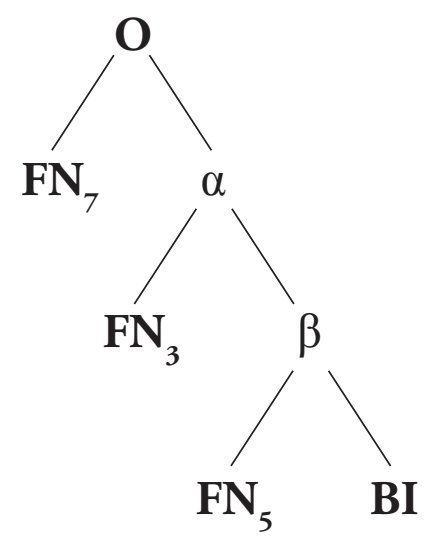

Come testo base utilizzo $\mathbf{F N}_{7}$ poichè si tratta di un manoscritto molto curato e di uno dei collettori di versi di Castiglione e Gonzaga.

Io dico spesso: «Amor, chi dà tal vena di pianto agli occhi miei che mai non manchi? Ond'è che ' 1 tristo cor mai non si stanchi in sì lunghi sospir e lunga pena?

Chi tanto gli alti miei desiri affrena e chi dapoi gli fa sì arditi e franchi? Chi dà vigore a' piedi afflitti e stanchi dietro al camin ch'a sua morte gli mena?» 
Ei mi risponde allhor: «Se ti nutrica, de l'alma luce sol degli occhi suoi, la dolce nostra e di pietà nemica,

in questi pur tu stesso veder pôi

— descritta di mia man, senza ch'io '1 dica la lunga storia degli affanni tuoi».

1 chi] che FN7

$\mathbf{2}$ di pianto] de pianto $\mathbf{F N} 3$, di pianti FN5

2 miei] mei FN3
8 camin] camminFN5

8 gli] li $\alpha$

9 Se] se'l $\alpha$ (con-1- cassata in FN5)

3 Ond'è ] Corretto su precedente Ond'ei FN5 9 ti] te FN3

3 si] se $\mathbf{F N} 3$

4 lunghi...lunga] longhi...longa FN3

$4 \mathrm{e}]$ sì $\beta$, sovrascritto a sì cancellato $\mathbf{F N} 7$

5 gli] gl'FN5, li FN3

5 miei] mei FN3

5 desiri] disiri $\beta$; desir FN3

6 gli] li $\alpha$

7 vigore] vigor FN3, BI

7 a'] ai FN5

8 dietro] drieto $\beta$

Università di Trento
10 suoi] soi $\mathrm{FN} 3$

11 e di] di $\beta$

11 pietà ] Corretto su precedente pietra FN7

12 questi pur ] questo sol $\beta$

$12 \mathrm{tu}]$ te $\beta$

12 pôi] puoi $\beta$

13 di] de FN3

13 ch'io'l] ch' io $\alpha$

$\mathbf{1 4}$ storia] historia FN3, BI

\section{Opere citate}

Nella seguente Bibliografia non sono elencate le stampe antiche né i testimoni a stampa delle rime di Castiglione e Gonzaga.

Arlsan, Edoardo, "Baldassarre Estense”, Dizionario Biografico degli Italiani, V (1963).

Balduino, Armando, Due sonetti inediti di Baldassar Castiglione, in Ventitré aneddoti raccolti nell'Istituto di Filologia e Letteratura italiana dell'Università di Padova, a cura di Ginetta Auzzas e Manlio Pastore Stocchi. Vicenza: Neri Pozza, 1980, pp. 33-39. 
- Due sonetti inediti di Baldassar Castiglione, in Periferie del petrarchismo, a cura di Beatrice Bartolomeo e Attilio Motta. Roma-Padova: Antenore, 2008, pp. 115-122.

Bandello, Matteo, Rime, a cura di Massimo Danzi. Modena: Edizioni Panini-Istituto di studi rinascimentali di Ferrara, 1989.

Bellorini, Giuliano, Il magnifico Signor Cavallier Luigi Cassola Piacentino. Edizione critica dei madrigali. Censimento e indice dei capoversi di tutte le rime. Firenze: Olschki, 2012.

. "Luigi Cassola madrigalista", Aevum, LXIX (1995): 593-615.

Bembo, Pietro, Le rime, a cura di Andrea Donnini, 2 voll. Roma: Salerno, 2008.

Bolzoni, Lina, Poesia e ritratto nel rinascimento. Roma-Bari: Laterza, 2008.

Brambilla Ageno, Franca, L'edizione critica dei testi volgari. Padova: Antenore, 1984.

Cassola, Luigi, Il canzoniere del codice Vaticano Capponiano 74, a cura di Giuliano Bellorini. Piacenza: Tip.Le.Co., 2002.

Castiglione, Baldassarre, La seconda redazione del "Cortegiano» di Baldassar Castiglione, a cura di Ghino Ghinassi. Firenze: Sansoni, 1968.

. Le lettere. 1497-marzo 1521, a cura di Guido La Rocca. Milano: Mondadori, 1978.

. Rime e giochi di corte, a cura di Michela Fantato, prefazione di Giancarlo Malacarne e Gilberto Pizzamiglio. Mantova: Universitas mercatorum Mantuæ (volume monografico dell'omonima rivista), 2004.

- Vita di Guidobaldo duca di Urbino, a cura di Ubaldo Motta. Roma: Salerno, 2006.

Cian, Vittorio, "Nel mondo di Baldassarre Castiglione", Archivio Storico Lombardo, VII (1942): 9-97.

. "Un codice ignoto di rime volgari appartenuto a B. Castiglione", Giornale storico della letteratura italiana, XXIV-XXV (1899-1900): 297-353, 53-93.

. Un illustre nunzio pontificio del rinascimento. Baldassar Castiglione. Città del Vaticano: Biblioteca Apostolica Vaticana, 1951.

Cisano, Giovanni, Tesoro di concetti poetici [...] ridotti sotto capi per ordine d'alfabeto da Giovanni Cisano [...], Venezia, Evangelista Devechino e Giovan Battista Pulciani, 1610, 2 voll.

Crescimbeni, Giovanni Mario, Istoria della volgar poesia. Roma: Chracas, 1698.

Del Castillo, Hernando, Cancionero general, a cura di Joaquín González Cuenca. Madrid: Editorial Castalia, 2004. 
Dionisotti, Carlo, "Recensione a Vittorio Cian, Un illustre nunzio pontificio", Giornale storico della letteratura italiana, CXXIX (1952): 67-83.

Fantato, Michela, "Baldassarre Castiglione. Rime e giochi di corte", Humanistica, III/1 (2008): 45-54.

Finazzi, Maria, Edizione critica delle rime del canzoniere di Ludovico Ariosto, tesi di dott., XVI ciclo, relatore prof. Simone Albonico, Università degli studi di Pavia, 2002-2003.

Flamini, Francesco, "Jacopo Corsi e Tebaldeo", Giornale storico della letteratura italiana, XVIII (1891): 395-396.

Giovio, Giovan Battista, Gli uomini della comasca diocesi nelle arti, e nelle lettere illustri. Bologna: Forni, 1975, ristampa anastatica dell'edizione Modena: Società Tipografica, 1784.

Gorni, Guglielmo, Repertorio metrico della canzone italiana dalla Origini al Cinquecento. (REMCI). Firenze: Franco Cesati, 2008.

Gorni, Guglielmo, Massimo Danzi e Silvia Longhi (a cura di), Poeti del Cinquecento. Poeti lirici burleschi satirici e didascalici. Milano-Napoli: Ricciardi, 2001.

Grasso, Nicola, Eutichia, a cura di Luigina Stefani. Messina-Firenze: D’Anna, 1978.

Kristeller, Paul Oskar (a cura di), Iter Italicum, 6 voll. London-Leiden: The Warburg Institute-E. J. Brill, 1967-1992.

Malacarne, Giancarlo, "Castiglione inedito. Rinvenuto un giacimento letterario da studiare e pubblicare", Universitas mercatorum Mantue, 15 (dicembre 2002): 4-7.

Marchand, Jean-Jacques, Strategia attributiva nella poesia cortigiana: il caso di Tebaldeo. Storia dell'arte, musicologia, letteratura, in L'attribuzione: teoria e pratica, a cura di Ottavio Besomi e Carlo Mancuso. Basel: Birkhäuser, 1994.

Morel-Fatio, Alfred, "A propos du sonnet Superbi colli”, Bulletin Italien, III (1903): 37-38.

. "Histoire d'un sonnet", Revue d'histoire litteraire de la France, I (1884): 97-101.

Motta, Uberto, Castiglione e il mito di Urbino. Studi sulla elaborazione del "Cortegiano». Milano: Vita e Pensiero, 2003.

- "Questioni testuali castiglionesche: attribuzione, tradizione, commento (con una glossa sulle «Rime» del Bembo)", Aevum, 81 (2007): 705-742.

Parenti, Giovanni, Introduzione, edizione, traduzione e commento a quattro carmina di Baldassar Castiglione, in Per Domenico De Robertis. Studi offerti dagli allievi fiorentini, a cura di Isabella Beccherucchi, Simone Giusti e Natascia Tonelli. Firenze: Le Lettere, 2000, pp. 345-397. 
- Per Castiglione latino, in Per Cesare Bozzetti. Studi di letteratura e filologia italiana, a cura di Simone Albonico, Andrea Comboni, Giorgio Panizza e Claudio Vela. Milano: Fondazione Arnoldo e Alberto Mondadori, 1996, pp. 185-218.

Poggiali, Niccolò, Memorie per la storia letteraria di Piacenza, 2 voll. Piacenza: Niccolò Orcesi, 1789.

Quadrio, Francesco Saverio, Della storia e della ragione d'ogni poesia, 4 voll. Bologna: F. Pisarri, 1739-1749.

Quondam, Amedeo, Petrarchismo mediato: per una critica della forma antologia. Roma: Bulzoni, 1974.

Rime d'amore di Baldassar Castiglione. Mantova: Grassi, 1974.

Salvadè, Anna Maria, Per un carme del Castiglione latino, in Imitar gli antichi. Appunti sul Castiglione. Milano: Unicopoli, 2006, pp. 11-32.

Scarpa, Emanuela, Per l'edizione di un poeta cinquecentesco: sulle rime di Giovanni Muzzarelli, in La critica del testo. Atti del convegno di Lecce, 22-26 ottobre 1984. Roma: Salerno, 1985, pp. 531-560.

Scarpati, Claudio e Uberto Motta, Studi su Baldassarre Castiglione. Milano: ISU_ Università Cattolica, 2002.

Stefani, Luigina, "Le «Ottave d'Italia» del Castiglione e le feste urbinati del 1513", Paragone, CCCXXXII (1977): 67-83.

Storia di Como scritta da Maurizio Monti professore nel liceo diocesano della stessa città, 2 voll. Como: Pietro Ostinelli, 1829-1832.

Stussi, Alfredo, Introduzione agli studi di filologia italiana. Bologna: il Mulino, 2003.

Vagni, Giacomo, "Su un sonetto di Ercole Strozzi già attribuito a Baldassar Castiglione", Aevum, LXXXV (2011): 751-775.

. "L'«honorata schiera» della duchessa Elisabetta. Ipotesi attributive sul Tirsi di Baldassar Castiglione e Cesare Gonzaga", «Aevum» LXXXVII (2013): 734-758.

- Una breve raccolta di liriche dedicata ad Elisabetta Gonzaga da Baldassar Castiglione e Cesare Gonzaga, in I Gonzaga e i papi. Roma e le corti padane fra Umanesimo e Rinascimento (1418-1620), a cura di Renata Salvarani. Città del Vaticano: Libreria Editrice Vaticana, 2014.

Vela, Claudio, Luigi Cassola e il madrigale cinquecentesco, in Tre studi sulla poesia per musica. Pavia: Aurora edizioni, 1984, pp. 29-65.

Vetrugno, Roberto, Baldassar Castiglione, in Autografi dei letterati italiani. Il Cinquecento, a cura di Matteo Motolese, Paolo Procaccioli e Emilio Russo. Roma: Salerno, 2009, pp. 135-147. 